\title{
Control of domain structure and magnetization reversal in thick Co/Pt multilayers
}

\author{
L. Fallarino, ${ }^{1}$ A. Oelschlägel, ${ }^{1}$ J. A. Arregi, ${ }^{2}$ A. Bashkatov ${ }^{3}$ F. Samad, ${ }^{4}$ B. Böhm, ${ }^{4}$ K. Chesnel, ${ }^{5}$ \\ and O. Hellwig ${ }^{1,4}$ \\ ${ }^{1}$ Helmholtz-Zentrum Dresden-Rossendorf, Institute of Ion Beam Physics and Materials Research, \\ Bautzner Landstrasse 400, 01328 Dresden, Germany \\ ${ }^{2}$ CEITEC BUT, Brno University of Technology, Purkyňova 123, 61200 Brno, Czechia \\ ${ }^{3}$ Institute of Fluid Dynamics, Helmholtz-Zentrum Dresden-Rossendorf, Dresden, Germany \\ ${ }^{4}$ Institute of Physics, Chemnitz University of Technology, D-09107 Chemnitz, Germany \\ ${ }^{5}$ Department of Physics and Astronomy, Brigham Young University, USA
}

submitted on October $13^{\text {th }}, 2018$, accepted for publication in Physical Review B (January $2^{\text {nd }}, 2019$ )

We present a study of the magnetic properties of $[\operatorname{Co}(3.0 \mathrm{~nm}) / \operatorname{Pt}(0.6 \mathrm{~nm})]_{N}$ multilayers as a function of $\mathrm{Co} / \mathrm{Pt}$ bilayer repetitions $N$. Magnetometry investigation reveals that samples with $N \geq$ 15 exhibit two characteristic magnetization reversal mechanisms, giving rise to two different morphologies of the remanent domain pattern. For applied magnetic field angles near the in-plane field orientation, the magnetization reversal proceeds via a spontaneous instability of the uniform magnetic state resulting in perpendicular stripe domains. Conversely, for field angles close to the out-of-plane orientation, the reversal occurs via domain nucleation and propagation leading to a maze-like domain pattern at remanence. Our measurements further enable the characterization of the $N$-dependent energy balance between the magnetic anisotropy and magnetostatic energy contributions, revealing a gradual disappearance of the domain nucleation process during magnetization reversal for $N<14$. This leads to the exclusive occurrence of an instability reversal mechanism for all field orientations as well as aligned-like stripe domains at remanence. Furthermore, a detailed study of the influence of the magnetic history allows the determination of a range of material properties and magnetic field strengths, where a lattice of bubble domains with remarkably high density is stabilized. These modulations of the ferromagnetic order parameter are found to strongly depend on $N$, in terms of center-to-center bubble distance as well as of bubble diameter. Moreover, such $\mathrm{Co} / \mathrm{Pt}$ multilayers could be utilized to engineer field reconfigurable bubble domain lattices, which can resemble magnonic crystals. 


\section{I.Introduction}

Magnetic thin films with competing long and short-range interactions are a very important research topic in the field of ferromagnetism [1,2]. Due to their close competition, complex magnetization reversal processes as well as spatially inhomogeneous magnetic multi-domain states generally occur [3-13]. Undoubtedly, artificial layered structures consisting of alternating ferromagnetic (FM) / non-magnetic layers rightfully belong to this category [14-18]. In these systems, magnetocrystalline and surface anisotropies are typically opposing the magnetostatic selfinteraction, causing the occurrence of heterogeneous microscopic magnetic states in order to minimize their total energy [17,19-21]. The resulting nanoscale magnetic textures have attracted significant interest due to their technological potential [22-28], as well as for their structural complexity [29-32]. In particular magnetic bubbles, which correspond to cylindrically shaped domains extending throughout the total thickness [33], were intensively studied largely motivated by their potential for applications in solid state storage [34,35]. While the industrial interest was pushed down by the subsequent advent of more efficient commercial devices [36], both the geometrical implication of magnetic bubble patterns and their dynamic behavior have become to a greater extent their primary research aspect [37-39]. As a matter of fact, a dipolar-stabilized bubble domain might be considered as a topological spin texture alike chiral skyrmions [40]. However, besides possible domain wall defects leading to achiral bubbles, the symmetric nature of dipolar interactions would lead to the co-existence of equal amounts of bubbles with both chiralities without the application of advanced patterning techniques together with specific magnetic field treatments [41].

Interestingly, by specific material parameter choices, the uniform ferromagnetic ground state can be established in artificial layered structures [42-44]. Therefore, a key issue in multilayer films is to find out under which conditions the system prefers spatial modulations of its FM order parameter instead of a simple homogeneous magnetic ground state, as well as to understand the mechanism governing the associated modulation period. The specifics of the resulting micromagnetic states are set by the relative strength of the competing interactions, whose ratio in multilayer structures can be tuned by changing for instance the individual layer thicknesses or the number of layer repetitions [17,45-47]. Moreover, while the energy balance is fixed by the material parameters, it was shown by Gao et al. [48] that the remanent magnetic domain state configuration 
can be efficiently manipulated by applying an appropriate magnetic field routine leading to a highly dense remanent bubble domain lattice $[49,50]$.

In the last two decades, an extensive work effort has led to an apparently very complete understanding of magnetization textures in magnetic multilayers [16,17,51,52]. The majority of these studies, though, utilized very thin FM layers, since an in-plane magnetization reorientation is expected for thicker films [47,53-55]. However, for sufficiently thick FM layers and in the presence of out-of-plane crystalline magnetic anisotropy, it was found that the magnetization undergoes a second reorientation transition back to out-of-plane orientation $[6,12,13,30,56,57]$.

Based on the prior knowledge of FM multilayer thin films and our observations of various magnetic domain morphologies depending on magnetic history $[49,50]$, the purpose of this paper is to provide an extensive exploration and description of their magnetization reversal as a function of the energy balance between magnetostatic and anisotropy energies. More importantly, the aim is to determine the existence of a specific energy ratio able to enhance the domain densities at remanence $[48,49]$ and in particular possibly stabilizing dense arrays of magnetic bubbles. This has been carried out by optimizing the total thickness of $[\mathrm{Co}(3.0 \mathrm{~nm}) / \operatorname{Pt}(0.6 \mathrm{~nm})]_{N}$ multilayers by varying the $\mathrm{Co} / \mathrm{Pt}$ bilayer repetitions $N$ in a range unexplored heretofore, in combination with finely adjusting the magnitude of the previously applied magnetic field.

Among the elemental ferromagnets, bulk Co adopts at room temperature the hexagonal close packed ( $h c p)$ crystal structure and possesses a magnetic easy axis (EA) along the $c$-axis. Thus, we have grown our multilayer films on top of a thick Pt (111) buffer layer such that Co grows with the necessary texture to induce an out-of-plane anisotropy-axis orientation. By studying in detail the influence of the magnetic history on the remanent domain pattern, and after having determined an optimal Co thickness leading to the formation of a bubble state [49], we find an optimal $N$ for which a dense lattice of bubble domains is favored and the bubble density is maximized.

The paper is organized as follows. We describe the utilized methods in Section II. Then, in Section III A, the identification of the crystal structure and the evaluation of the epitaxial relationships are shown. In Section III B, the room temperature magnetometry characterization is presented and analyzed. The remanent magnetic states for various $N$ including a magnetic domain density study as a function of the previously applied magnetic field are shown and discussed in Section III C-1. Section III C-2 describes the remanent magnetic state diagram of such multilayer structures in terms of color coded-maps of the domain density with respect to external parameters. 
Finally, Section IV provides a summary of the accomplished results and general conclusions that can be drawn from our work.

\section{II.Methods}

The $[\mathrm{Co}(3.0 \mathrm{~nm}) / \mathrm{Pt}(0.6 \mathrm{~nm})]_{N}$ multilayer $(\mathrm{ML})$ films were prepared at room temperature by dc magnetron sputter deposition in an ultrahigh vacuum (UHV) system (ATC 2200 series from AJA International, Inc.) with a base pressure better than $3 \times 10^{-6} \mathrm{~Pa}$. Si substrates covered by a $100 \mathrm{~nm}$ thick thermal $\mathrm{SiOx}$ layer were used. For each layer, the deposition process was started only after pre-sputtering the targets for at least $30 \mathrm{~s}$, using a pressure of $4 \times 10^{-1} \mathrm{~Pa}$ of pure Ar atmosphere. As a template for the growth of (0001) textured hcp Co layers, a $1.5 \mathrm{~nm}$ Ta and subsequent $20 \mathrm{~nm}$ Pt layer were deposited using plasma power settings of $100 \mathrm{~W}$ and $30 \mathrm{~W}$ respectively. Thereafter, the Co and Pt depositions were performed by using $80 \mathrm{~W}$ and $30 \mathrm{~W}$ plasma powers respectively. Each sample was finally coated with a $2.3 \mathrm{~nm}$ thick Pt layer to avoid surface oxidation and contamination after removal from the vacuum system as well as aging effects. The Pt was also chosen as capping material to avoid breaking the spatial inversion symmetry along the out-of-plane (OOP) direction of the multilayer structure [58]. A schematic of the sample structure, including its specific layer sequence, is shown in Fig. 1(a) (top right part) together with the corresponding thicknesses. The structural analysis of the samples was performed by means of $\mathrm{X}$-ray diffraction (XRD) and reflection (XRR) utilizing a Rigaku SmartLab x-ray Diffractometer with $\mathrm{Cu}-\mathrm{K} \alpha$ radiation. Magnetization measurements were performed using a commercial Microsense EZ7 vibrating sample magnetometer (VSM), equipped with a $360^{\circ}$ rotational stage. Finally, magnetic domain imaging was performed via magnetic force microscopy (MFM) using a Bruker Multimode IV atomic force microscope and NSC18/Co-Cr/Al BS magnetic tips.

\section{III.Results and Discussion}

\section{A. Structural characterization}

In order to characterize the periodicity of the compositional modulation, we studied the crystallographic structure and the OOP mosaicity, for which XRR as well as XRD $\theta-2 \theta$ scans and rocking curve measurements were performed. Fig. 1(a) illustrates XRR $\omega-2 \theta$ scans in the angular range $0.2^{\circ} \leq 2 \theta \leq 9.0^{\circ}$ for the entire set of samples investigated in this study. Each measurement has been normalized to its maximum intensity and vertically offset by a constant value to permit 
direct comparison. Interference-caused Kiessig oscillations with two different wavelengths are distinguishable in Fig. 1(a). A first type can be observed at low $2 \theta$ values, whose period is inversely proportional to the number of $\mathrm{Co} / \mathrm{Pt}$ repetitions $N$. These short-wavelength oscillations correspond to the total thickness of the multilayers, since by increasing $N$ (i.e. the total sample thickness) the distance in between two consecutive minima or maxima decreases. At the same time their relative intensity decreases while increasing $N$, due to the increasing number of interfaces as well as the absorption in each individual layer (whose number is $2 N$ ). In a wider $2 \theta$-range a second set of Kiessig oscillations is noticeable, whose period $\left(\Delta \theta \approx 0.2^{\circ}\right)$ is constant as a function of $N$. They originate from the $20 \mathrm{~nm}$ thick Pt buffer layer, whose thickness is set to be constant independently of the sample. More importantly first and second order Bragg-like superstructure peaks, appearing at $2 \theta \approx 2.55^{\circ}$ and $2 \theta \approx 4.80^{\circ}$ respectively, have been measured for the entire set of our samples, giving a clear signature of a well-defined periodic elemental modulation of the multilayer structure independent of $N$. While it may be expected to observe superstructure peaks up to higher orders, the interfaces of any real multilayer system present slight imperfections due to roughness and interdiffusion. Such small deviations from perfect interfaces are partly responsible for the rapid reflectivity reduction of the intensities especially for higher order superstructure peaks as well as for the absolute broadening of their width. Moreover, due to the selected thickness of the individual layers, the higher order peaks are expected to be located at high angles $2 \theta$ where the reflected xray intensity has decreased rapidly with the inverse fourth power of the transferred wave vector magnitude [59]. Furthermore, the broadening of the individual ML-Bragg peaks is $N$-dependent: by increasing the number of $\mathrm{Co} / \mathrm{Pt}$ bilayer repetitions, the width of the ML-Bragg peaks decreases substantially, as can be seen in Fig. 1(a). While this is partly caused by the sharpening of interference maxima with increasing $N$ (analogously to the situation for diffraction gratings) as well as by the decreased overlap with Kiessig oscillations due to their reduced intensity and width, it can be furthermore notably linked to the stability of the $\mathrm{Co} / \mathrm{Pt}$ bilayer thickness. Indeed, while increasing the number of $\mathrm{Co} / \mathrm{Pt}$ bilayer blocks of constant total thickness $t_{C o+P t}$ within the experimental error, the individual $t_{C o+P t}$ should have a distribution getting closer and closer to a normal distribution where an angular broadening of the ML-Bragg peak decreases while increasing $N$ [59]. Therefore, the observed evolution of the $1^{\text {st }}$ and $2^{\text {nd }}$ order full width at half-maximum (FWHM) peak value is importantly confirming that the material parameters uniformity and reproducibility stay constant while increasing $N$. 
Figs. 1(b) and (c) display XRD $\theta-2 \theta$ scans in the angular range $22^{\circ} \leq 2 \theta \leq 102^{\circ}$ for all samples studied in this work, where the peak indexing in (c) refers also to (b). Similar to the measurements in Fig. 1(a), each dataset has been normalized to its Si (400) diffraction peak intensity and is vertically offset by a constant value for direct comparison. All the scans look nearly identical in their overall appearance, exhibiting only well-defined diffraction peaks corresponding to Si (400), buffer Pt (111) and (222), Co/Pt (0002)/(111) and (0004)/(222) lattice planes ${ }^{1}$. The Pt buffer layer peaks look virtually the same for all samples, both in terms of angular position and FWHM, verifying the robustness of our fabrication process. This template layer stability allows us afterwards to ascribe any significant change in the magnetic properties to the specific number of repetitions $N$ itself as opposed to inherent structural variations. Most relevantly, the entire set of samples exhibits a crystallographic orientation quality that is very similar to samples with thinner individual Co layers, despite the high total thickness and high number of repetitions of the $\mathrm{Co} / \mathrm{Pt}$ bilayer of the present study [46]. Thus, the total angular range shows only well-defined $f c c$ ( $n n n)$ and $h c p(000 l)$ peaks for Pt and Co. The $h c p$ lattice of Co was additionally verified via in-plane (IP) diffraction measurements, by setting the incident and diffracted beams nearly parallel to the sample surface, in which only Co $(11 \overline{2} 0)$ and (1010) reflections were measured together with the one of $\mathrm{Pt}$ (220) for any sample studied in this work ${ }^{2}$.

Moreover, satellite peaks of the multilayer diffraction signals [indexed by $n$ in Fig. 1(c)] have been measured, which are a clear indication of a perpendicular structural and material coherence far greater than the thickness of the individual layers. It can be also observed that the negative indexed satellite diffraction peaks have higher intensity than the positive ones, with the latter being within the noise level for $n>1$. However, this effect is mainly caused by the overlapping and interfering waves coming from the $-n$ satellite diffractions and the Pt (111) and Pt (222) diffraction planes. By using a triple Gaussian fitting function ${ }^{3}$, the positions of the $1^{\text {st }}$ and $2^{\text {nd }}$ order low angle Bragg-like superstructure peaks, displayed in Fig. 1(a), were evaluated in order to estimate the average total thickness of the Co/Pt bilayer $\bar{t}_{\mathrm{Co}+\mathrm{Pt}}{ }^{4}$. Fig. 2(a) shows the $N$-dependence

\footnotetext{
${ }^{1}$ In Fig. 1(c) and in the text Co/Pt* refers to Co (0002)/ Pt (111), whereas Co/Pt** to Co (0004) / Pt (222).

${ }^{2}$ However, our XRD-investigation cannot exclude the presence of $f c c$ stacking faults.

${ }^{3}$ It was used a triple Gaussian function in order to take into account the superposition of left and right Kiessig oscillations coming from the total thickness and Pt buffer layer on the $1^{\text {st }}$ and $2^{\text {nd }}$ order Bragg peaks respectively.

${ }^{4}$ Here, the average value refers to two independent averaging processes: one is directly connected to the volume averaged nature of the x-ray measurements, which is indeed dependent on the penetration depth of the beam as well
} 
of $\bar{t}_{\mathrm{Co}+\mathrm{Pt}}$, where the errorbars correspond to the standard deviation values. Under the assumption of a purely statistical Gaussian distribution for the observed $\bar{t}_{\mathrm{Co}+\mathrm{Pt}}$ values, all data fall into the interval defined by $\overline{\bar{t}}_{\mathrm{Co}+\mathrm{Pt}} \pm \sigma=3.61 \pm 0.01 \mathrm{~nm}$, with $\sigma$ being the standard deviation from the data average $\overline{\bar{t}}_{\mathrm{Co}+\mathrm{Pt}}$. Indeed, the experimentally determined $\overline{\bar{t}}_{\mathrm{Co}+\mathrm{Pt}}$ is consistent within the statistically estimated error with the nominal total thickness value of $3.6 \mathrm{~nm}$.

The average lattice constant of the $\mathrm{Co} / \mathrm{Pt}$ bilayer was extracted from the angular positions of the $\mathrm{Co} / \mathrm{Pt}^{*}, 1$ diffraction peaks in the $\theta-2 \theta$ scans, and plotted in Fig. 2(b) as a function of $N$ together with the associated errors estimated from the least-squares Gaussian fit. The data are displayed along with reference values for bulk Pt (blue dashed line) and Co (red dotted line), for comparison. The lattice constants $\bar{d}_{\mathrm{Co}+\mathrm{Pt}}$ shown in Fig. 2(b) correspond to the OOP interplanar distance of the superlattice-like cell consisting of both Co and Pt. Besides the absence of notable variations in between the extracted data, the values are consistently and substantially smaller than what one would expect for a Pt-rich superlattice cell or pure bulk Pt. The experimentally determined average value of $\overline{\bar{d}}_{\mathrm{Co}+\mathrm{Pt}}=0.2089 \mathrm{~nm}$ is only slightly larger than the corresponding weighted average thickness of $0.208 \mathrm{~nm}$ that is extracted from the bulk parameters of the $\mathrm{Co} / \mathrm{Pt}$ multilayer structure parameters in this work. Furthermore, Fig. 2(b) reveals a slight trend towards smaller $\bar{d}_{\mathrm{Co}+\mathrm{Pt}}$ values as $N$ is increased. This effect could be caused by the initial strain from the 20 $\mathrm{nm}$ thick Pt buffer layer, which is stronger for less $N$.

In order to investigate the OOP crystallographic axis dispersion in the samples, rocking curve measurements have been performed for the $\mathrm{Pt}(111)$ and $\mathrm{Co} / \mathrm{Pt}^{*}$ peaks ${ }^{1}$. The full widths at half maximum values of the peaks are plotted in Figs. 2(c) and (d) as a function of $N$, with the error-bars representing uncertainties estimated via the least-square Gaussian fits. Both sets of measurements indicate that the samples achieved remarkably good alignment of $\mathrm{Co} / \mathrm{Pt}$ crystallographic $c$-axis orientation normal to the sample surface, when compared to previous works $[60,61]$. Finally, our structural sample analysis confirms the good crystallographic quality of the optimized layer growth sequence resulting in well-modulated $\mathrm{Co} / \mathrm{Pt}$ multilayer films with perpendicular $c$-axis orientation, necessary for a preferential OOP orientation of the magnetization in such thick structures.

as on its lateral extension; the second one is the arithmetic mean of the thicknesses calculated via the angular position of the $1^{\text {st }}$ and $2^{\text {nd }}$ order Bragg-like superstructure peaks at low angles. 


\section{B. Magnetic characterization}

The macroscopic magnetic properties were analyzed to verify whether and how the designed multilayer structures exhibit a preferential orientation of their magnetization $M$ parallel to the film normal. Figs. 3(a)-(m) present room temperature normalized $M / M_{S}$ data as a function of the field strength $\mu_{0} H$ and number of $\mathrm{Co} / \mathrm{Pt}$ bilayer repetitions $N$, with $M_{S}$ being the saturation magnetization. For all hysteresis loops shown here, the (black) short dashed and (red) solid lines show the magnetization curves measured for an external magnetic field applied parallel and perpendicular to the film plane respectively. Fig. 3(a) shows the data for the thinnest sample in this study with $N=6$. Despite the OOP orientation of the magnetocrystalline anisotropy axis, an inplane behavior was found. The measurement shows an almost perfectly rectangular-shaped hysteresis for the IP applied field with an abrupt magnetization reversal. In contrast, the OOP field data in Fig. 3(a) show an almost completely reversible change in the magnetization orientation where the complete alignment is reached only at a field strength of $\mu_{0} \mathrm{H}_{\mathrm{S}}^{\mathrm{OOP}} \approx 1.3 \mathrm{~T}$. The small hysteresis effect during the OOP reversal process is the result of slight sample imperfections. At the other end of our sample spectrum, Fig. $3(\mathrm{~m})$ shows two reversal curves that are a clear indication of OOP preferential orientation of the magnetization $[6,7,17]$. For $\mu_{0}|\mathrm{H}|>\mu_{0} \mathrm{H}_{\mathrm{S}}^{\mathrm{OOP}} \approx 1.15$ $\mathrm{T}$, the system exhibits in both geometries a uniform magnetization state parallel to the field direction. In the IP field case the saturated state becomes unstable at the critical field $\mu_{0}\left|\mathrm{H}_{\text {cr }}\right| \approx 0.5$ $\mathrm{T}$ upon reducing the field strength, and undergoes laterally alternating magnetization rotations driven by the magnetocrystalline OOP anisotropy culminating in the formation of a remanent stripe domain configuration. However, an IP magnetization component persists at remanence mainly within the domain walls, which is aligned during the field sequence and which is responsible for the hysteresis that occurs for low field values [6-8]. For the OOP field configuration, once the field is lowered, the uniform state is altered by the formation of bubble domains with opposite magnetization orientation driven by magnetostatic energy. This process starts rather abruptly at the nucleation field $\mu_{0}\left|\mathrm{H}_{\mathrm{n}}^{\mathrm{OOP}}\right| \approx 1 \mathrm{~T}$, leading to a sharp drop in the magnetization. As the field is further reduced the domain dimensions increase resulting in the linear field dependence of the magnetization down to remanence. The observed small hysteresis effect is again the result of slight sample imperfections [62]. 
From our experimental data in Fig. 3, we concluded that $N$ has a profound impact on the magnetization reversal characteristics of thick $\mathrm{Co} / \mathrm{Pt}$ multilayers. While the overall appearance of the IP and OOP magnetization reversal curves stays very similar for $16 \leq N \leq 30$ [Figs. 3(f)-(m)], an appreciable change occurs in the two OOP-hysteretic regions at high field magnitudes, associated with the nucleation and annihilation of domains. In fact, upon increasing $N$, their position along the magnetic field axis shifts to progressively higher values as well as their area becomes gradually larger. This is evidenced in the close-up plots shown in Figs. 3(n)-(q). Differently, by decreasing $N$ below 14, these hysteretic areas collapse entirely as displayed in Fig. 3(d). In contrast to this change in the nucleation behavior, the central hysteresis does not disappear, but instead actually increases significantly between $N=14$ and $N=10$ for OOP field orientation, before it decreases again for even smaller $N$, consistent with previous observations in pure Co systems when decreasing the effective OOP anisotropy [6,13]. Also, the saturation magnetization maintains a nearly constant value of $\bar{M}_{\mathrm{S}}=1147 \pm 25 \mathrm{kA} / \mathrm{m}$ (see Supplemental Material [63]) in the entire $N$-range studied. The OOP saturation field $\mu_{0} \mathrm{H}_{\mathrm{S}}^{\mathrm{OOP}}$, the nucleation field $\mu_{0} \mathrm{H}_{\mathrm{n}}^{\mathrm{OOP}}$, and the IP saturation field $\mu_{0} \mathrm{H}_{\mathrm{S}}^{\mathrm{IP}}$ strengths are plotted as a function of $N$ in Figs. $3(\mathrm{r})$ and (s). The $\mu_{0} \mathrm{H}_{\mathrm{S}}^{\mathrm{OOP}}(N)$ data can be divided into two branches that join in a cusp-like point located at $N=14$. For $N \geq 14$ the OOP saturation field monotonically increases while increasing $N$ and hence the sample thickness, as already predicted by Thiele [33] and experimentally measured by Hehn et al. [7] for a single magnetic film with OOP anisotropy. On the contrary, for $N<14$, the preferred orientation of the magnetic moments at remanence changes from OOP to tilted and finally IP orientation, which is reflected in the monotonic increase of $\mu_{0} \mathrm{H}_{\mathrm{S}}^{\mathrm{OOP}}$ when reducing $N$.

Based on the behavior of the OOP loops and corresponding saturation fields, three different $N$-ranges can be identified in which the magnetic state before OOP saturation and the mechanism for reaching the saturated single domain state at $\mu_{0} \mathrm{H}_{\mathrm{S}}^{\mathrm{OOP}}$ are fundamentally different: (i) for $N>14$, before OOP saturation the sample consists of magnetic bubbles which collapse upon reaching $\mu_{0} \mathrm{H}_{\mathrm{S}}^{\mathrm{OOP}}$; (ii) in the interval $10 \leq N<14, \mu_{0} \mathrm{H}_{\mathrm{S}}^{\mathrm{OOP}}$ describes the field at which the laterally alternating non-uniform magnetization state vanishes in favor of a uniform magnetic state; (iii) and for $N<$ 10, reaching OOP saturation refers to the process during which the magnetization vector continuously rotates from the easy plane direction towards the OOP field direction. In contrast to $\mu_{0} \mathrm{H}_{\mathrm{S}}^{\mathrm{OOP}}$, both $\mu_{0} \mathrm{H}_{\mathrm{S}}^{\mathrm{IP}}$ and $\mu_{0} \mathrm{H}_{\mathrm{n}}^{\mathrm{OOP}}$ increase monotonically with $N$. The $\mu_{0} \mathrm{H}_{\mathrm{S}}^{\mathrm{IP}}$ evolution is mainly 
driven by the thickness-induced increase of the OOP effective anisotropy while increasing the total thickness of the investigated samples, as already observed in previous studies on single thick Co layer [6,7] (see also Supplemental Material [63] for a quantitative evaluation). The $N$-dependence of $\mu_{0} \mathrm{H}_{\mathrm{n}}^{\mathrm{OOP}}$ is directly related to the total thickness of the systems, for the nucleation field being a growing function of the film thickness [6,33].

As seen in Fig. 3, our multilayer samples show fundamentally different magnetization reversal processes while applying the magnetic field along in- and out-of-plane directions and by varying $N$. As a result, in order to fully magnetically characterize this kind of system, not only IP and OOP orientations of the magnetic field should be explored, but also any intermediate field angles. In order to perform this angular dependent study, a convenient methodology introduced in earlier studies was utilized [12,13], which takes advantage of the normalized magnetization difference $\Delta M / M_{S}$ between the ascending and descending branches of the magnetization curves. Correspondingly, $M(\mathrm{H})$ curves have been measured for different applied field orientations ${ }^{5} \beta$, in steps of $\Delta \beta=5^{\circ}$ for $30^{\circ} \leq \beta \leq 95^{\circ}$, and $\Delta \beta=2^{\circ}$ for $-30^{\circ} \leq \beta \leq 28^{\circ}$. The complete angular dependence of the normalized magnetization difference is shown in Fig. 4 as color-coded maps of $\Delta M / M_{S}(\beta, H)$ in the $8 \leq N \leq 30 \mathrm{Co} / \mathrm{Pt}$ bilayer repetitions range ${ }^{6}$. As it can be clearly seen, the resulting $\Delta M / M_{S}$ $(\beta, \mathrm{H})$ maps strongly depend on $N$, showing significant changes especially in the high magnetic field regime. For $N=30$, the low magnetic field hysteresis forms a central band that extends from left to right over all magnetic field angles, even though it decreases in width near $\beta=0^{\circ}$. In contrast, the domain nucleation hysteresis is visible in this plot via the presence of two non-zero $\Delta M / M_{S}$ value regions centered at $\mu_{0} H \approx \pm 1 \mathrm{~T}$ and $\beta=0^{\circ}$. Upon changing $\beta$ away from the OOP orientation, the width of these regions in field gradually reduces, and they completely disappear for $\beta>10^{\circ}$ or $\beta<-10^{\circ}$. Hence we can conclude that for orientation $\beta>10^{\circ}$ and $\beta<-10^{\circ}$ the magnetization reverses by undergoing a second-order rather than a first-order phase transition $[12,13]$. On the contrary, the color-coded map for $N=8$ (Fig. 4) is characterized by the sole existence of a central hysteresis band, showing a slight increase near the OOP field orientation $\left(\beta=0^{\circ}\right)$. Therefore, we see that by varying the total magnetic thickness of the multilayer system, there is a threshold minimum total magnetic thickness $\left(N \times \mathrm{t}_{\mathrm{Co}}\right)$ for which hysteretic nucleation (and annihilation) of

\footnotetext{
${ }^{5} \beta=0^{\circ}$ corresponds to $H$ applied along the surface normal, $\beta=90^{\circ}$ refers to in-plane field orientation.

${ }^{6}$ The sample $N=6$ is not further investigated due to its similarity with respect to $N=8$.
} 
perpendicular bubbles and stripe domains occurs, a process indicated by the existence of non-zero $\Delta M / M_{S}$ regions outside the central band and near the OOP field orientation. Without much changing in field position, their angular extension shrinks progressively upon decreasing $N$, down to the point where they collapse entirely. In contrast to this change in the nucleation behavior, the central hysteresis band does not disappear, but instead increases. Analogous to the situation earlier observed in thick (0001) oriented Co-films [12,13], the angular extension of the nucleation process is controlled by the evolution of the $\mu_{0} \mathrm{H}_{\mathrm{cr}}(\beta)$ and $\mu_{0} \mathrm{H}_{\mathrm{n}}(\beta)$ curves and by their complete different angular dependence that produces a crossing at a specific $\beta$. By reducing the ratio between anisotropy and magnetostatic ${ }^{7}$ energy in favor of the latter, which was achieved for instance in Ref. 13 by increasing the temperature of pure single Co films, the angular position of the crossing point shifts towards $\beta=0^{\circ}$ until its complete suppression. Thus the experimental observations in Fig. 4 reflect the thickness-induced variations of the $\left(\beta, \mu_{0} H\right)$ regions where each of the two magnetization reversal mechanisms dominate for our $\mathrm{Co} / \mathrm{Pt}$ multilayers, as a result of the inherent thickness dependent ratio of magnetic anisotropy and magnetostatic energies. In fact by reducing $N$ (i.e. the total magnetic thickness), the crossing point between the two reversal mechanisms is gradually shifting to lower $\beta$ values until disappearing, which implies that the instability reversal process then occurs for any angle of the externally applied field.

In order to better visualize the energy-ratio dependence of the reversal mechanism, the same datasets shown in Fig. 4 were plotted and magnified for the extreme cases of $\beta=0^{\circ}$ and $90^{\circ}$ (OOP and IP, respectively), displayed in Fig. 5. For the OOP geometry [Fig. 5(a)] we can recognize two characteristics bands of non-zero $\Delta M / M_{S}$ values near applied fields of $\pm 1 \mathrm{~T}$ for high $N$ values. As $N$ is decreased from 30, an initial gradual reduction in field position of the two nucleation regions is followed by their complete disappearance for $N<14$. Differently, the central band stays nearly constant down to $N=14$, where it starts to expand significantly before shrinking again at the lowest $\mathrm{Co} / \mathrm{Pt}$ bilayer repetition used in our experiments. For the IP configuration, Fig. 5(b), a gradual reduction of the central hysteretic band with decreasing $N$ is visible without the appearance of any other hysteretic structure in the entire $\mathrm{N}$-range. This behavior is fully consistent with the high field hysteresis-free phase transition from a uniform state into an instability induced stripe domain

\footnotetext{
${ }^{7}$ Here and in the rest of the manuscript this term should not be mistaken with the shape anisotropy (i.e. the magnetostatic energy density of a uniform OOP state), but it refers to the magnetostatic energy density of the domain state instead.
} 
pattern (except for the lowest $N$, see below). This leads to a hysteresis peak around zero field due to the existence of a net IP magnetization component (partly within the domain walls) for this state. To conclude, by associating the existence of the high field hysteretic behavior with nucleation and the absence of it with instability-driven stripe domain generation, Fig. 5 implies that for $N<14$ the latter extends to every applied magnetic field angle.

However, an important aspect has still been neglected in our discussion. While lowering the number of bilayer repetitions from $N=30$, our samples are also experiencing a thicknessinduced magnetization reorientation transition that culminates at the lowest studied $N$-value with a reversal mechanism characterized by IP magnetization states alone $[6,30,57]$. In fact, the IP magnetization reversal curves displayed in Figs. 3(a) and (b) have lost the strong curvature of the high $N$-samples loops and exhibit almost full remanent magnetization. Thus, the samples with $N \leq$ 8 are evidently in an IP magnetization state at remanence, as it will be seen and further discussed in conjunction with Figs. 6 and 7. Importantly, the magnetization reversal evolution as a function of $N$ must have a direct impact on the remanent magnetic domain state for those samples with high repetition number $N$, enabling its manipulation in terms of modulation period and microstructure by applying specific magnetic field sequences. Therefore, character and tunability of the remanent magnetic domain structures have been investigated via MFM measurements in the next section.

\section{MFM characterization}

\section{Characteristic domain patterns}

The magnetometry characterization illustrates that qualitatively different magnetization reversal behaviors can emerge from the different balance between the magnetic anisotropy and magnetostatic energy contributions, which can be tuned in our thick multilayer films by means of varying $N$. The absence of the nucleation phase at low $N$ was identified across the entire $\mathrm{H}-\beta$ parameter space upon analyzing the color-coded maps in Figs. 4 and 5 [13]. In order to confirm the physical picture deduced from our VSM data, the remanent domain patterns were measured via MFM. For samples with $8<N<14$, we aim towards validating the persistence of a sufficiently large OOP anisotropy able to support a stripe domain state at remanence, despite the disappearance of the nucleation type hysteresis earlier found. For this purpose, the magnetic configuration was brought as close as possible to the equilibrium state in the OOP reference system by demagnetizing it, i.e. by applying an oscillating field of decreasing amplitude starting above $\mu_{0} \mathrm{H}_{\mathrm{S}}^{\mathrm{OOP}}$. Fig. 6 shows 
the corresponding remanent MFM images recorded after the described OOP demagnetization process as a function of $N$. At large thicknesses $(N \geq 18)$, the magnetic configuration consists of a disordered array of stripes with well-defined widths. The corresponding maze-like domain pattern is the result of the heterogeneous nucleation and expansion of bubble domains, which develops randomly due to the symmetry breaking caused by the nucleation process at $\mu_{0} \mathrm{H}_{\mathrm{n}}^{\mathrm{OOP}}$. Opposite to this, when looking at the MFM images for lower multilayer thicknesses $(10 \leq N \leq 16)$, the magnetic pattern is more ordered possessing a well-defined domain period. Indeed, for $N=10$, the perpendicular magnetization component is still found to have a periodic OOP up and down magnetization modulation, as confirmed by the weakly striped MFM contrast. Even though the IP component of the magnetization is coherently aligned along the stripes axis, it should not have in principle a preferential direction (opposite to the case of a film with IP uniaxial anisotropy $[64,65]$ ), because the stripe orientation is not fixed but instead depends on the sample history. However, for our samples with small $N$ values, a preferential direction along which the stripes align can be readily observed. We suggest that this is mainly triggered by the gradual extension to any magnetic field application angle $\beta$ of the instability-driven stripe domain generation, suppressing any level of disorder that the nucleation of bubbles may introduce (that for $N=14$ and 16 occurs only for $-2^{\circ} \leq$ $\beta \leq 2^{\circ}$ as depicted in Fig 4). Therefore, the whole stripe pattern orders parallel to the last experienced saturation field or, as in this case, to the direction of the small IP field component arising from possible experimental misalignment between the direction of application of $\mu_{0} \mathrm{H}$ and the sample surface normal. This attributes an oriented and "rotatable" IP anisotropy to the multilayer films with low $N$ despite OOP application of $\mu_{0} \mathrm{H}$ [66]: by changing the misalignment angle of the externally applied magnetic field during the demagnetization process, the small IP component would rotate accordingly on the sample surface plane. This induces a preferential axis for the stripe domains, due to the tilt of the stripe domain magnetization in the direction of the fields IP component and the associated Zeeman energy gain for an alignment of the stripes in this direction. Note that this behavior is not observed in samples with high $N$, due to the higher effective OOP anisotropy respectively in-plane saturation field, leading to a much less tilted magnetization in an external field with small IP component. Finally, as the magnetization curves obtained for $N$ $=6$ and $N=8$ are characteristic for a film with IP magnetization, the corresponding MFM image for $N=8$ displayed in Fig. 6 shows large IP domains with typical widths over $1 \mu \mathrm{m}$. A twodimensional Fast Fourier Transformation (2D-FFT) analysis taken over each large scan-size MFM 
images $\left(10 \times 10 \mu \mathrm{m}^{2}\right)$ confirms the $N$-dependent directionality of the stripe domains alignment. Decreasing the number of $\mathrm{Co} / \mathrm{Pt}$ repetitions $N$ causes the symmetric circle-like high intensity region gradually to break into two separated "quarter moon-like" structures.

In addition, we have studied the remanent domain patterns after IP demagnetization process. Fig. 7(a) and (b) shows MFM images for samples with $N=30$ and $N=10$ respectively. The alignment of the parallel component of the magnetization with the field, in order to minimize the Zeeman energy, leads to periodic patterns of parallel stripes for all samples between $10 \leq N \leq$ 30 , as already predicted and confirmed experimentally for systems with similar total thicknesses $[6,67]$. Although the investigated systems have a complicated magnetic behavior, the stripe domain state is generally accepted as the energetically preferred state in the absence of an applied magnetic field. As visible in Figs. 7(a) and (b) the symmetry breaking along the IP direction forces the stripe walls and stripes themselves to align along this direction. This is in contrast to the case of OOPdemagnetization (Fig.6) where, for samples exhibiting nucleation hysteresis, only the local order is preserved and the free-energy minimization as well as the finite temperature at which the experiment has been carried out lead to globally disordered patterns. However, we have seen that the disorder is suppressed while reducing the ratio between anisotropies and magnetostatic domain state energies as shown in Fig. 6 for $10 \leq N \leq 14$ : in this specific $N$-range the remanent domain state shows an aligned stripe domain pattern independent of the alternating magnetic field orientation, where the alignment is along the small IP field component present during the demagnetization process (except in the case of a virtually perfect OOP field alignment, which practically never occurs).

In order to evaluate the dependency of the domain size on $N$ (or on the total magnetic thickness), 2D-FFT of the MFM images after an IP demagnetization process were calculated using periodic boundary conditions $[11,66]$. Linear profiles crossing $k(0,0)$ were then extracted in order to evaluate the domain periodicity $\lambda$, with the spatial frequency $k o\left(\mu \mathrm{m}^{-1}\right)$ being defined as the center of the fit to a Gaussian curve as well the deviation $\Delta k_{0}\left(\mu \mathrm{m}^{-1}\right)$ in terms of its FWHM. Fig. 7(c) displays the domain periods $\lambda$ as a function of $\mathrm{Co} / \mathrm{Pt}$ bilayer repetition, where $\lambda$ increases for as $\propto \sqrt{N}$ in accordance with Kittel's law for stripe domains [2,30]. The red line in Fig. 7(c) corresponds to the calculated stripe domain period $\lambda$ by using the analytical expression developed in $[6,31,69,72]$ (see Supplemental Material [63]). 
An additional feature that is visible in Figs. 6 and 7 consists in that the MFM domain contrast is also $N$-dependent, dramatically decreasing for low $N$. This effect is mainly driven by the canting of the local magnetization induced by the IP reorientation for low $N$. Such a canting angle varies monotonically with $N$ as indicated by the $N$-dependence of the in-plane remanence ratio $M_{r}^{I P} / M_{S}$ shown in Fig. 7(d) (obtained from the magnetometry data of Fig.3). Samples with $N>20$ exhibit a low $M_{r}^{I P}$, arising mainly from the local magnetic moments confined inside the domain walls. The IP remanence gradually increases when lowering $N$ in the intermediate range $(10 \leq N \leq$ 20 ), indicating a canting of the domain magnetization towards the film plane. This behavior is in agreement with the appearance of the two low intensity regions along the otherwise high intensity rings into the 2D-FFT color-coded maps shown as insets in Fig. 6. The smaller domain size due to the reduction in total thickness [Fig. 7(c)] increases the number of domain walls, which however cannot account for the full increase of the IP remanent moment. Instead, it must be mainly caused by the increased tilting towards IP orientation while decreasing $N$. Finally, for $N<10$, the magnetization is fully in plane as indicated by the MFM images in Fig. 6. Similar observations of a thickness driven reorientation of the magnetization from IP to OOP (and vice versa) have been reported for numerous systems, including (0001) oriented Co films [5,6], polycrystalline alloy films [68,69], as well as in multilayer structures with ultrathin bilayer units [70,71]. There it was found that the magnetic behavior could be correctly described with the same values of saturation magnetization $M_{S}$, perpendicular anisotropy $K_{u}$ and exchange stiffness $A$ for each different sample of their series [5,6,31,68-73], and that the magnetic behavior was characteristic of weak perpendicular anisotropy materials with a $Q=2 K_{u} / \mu_{0} M_{S}^{2}$ smaller than 1 . Also in our study, we expect steadiness in these magnetic parameters, since the structural parameters stay constant (see Sec. III-A) and only $N$ is varied, yielding particularly the same crystalline and interface anisotropy. Indeed, quantitative evaluations of the hysteresis loops, stripe domain periods, as well as micromagnetic simulations, indicate that our whole sample series can be approximately described by the same values of $A, M_{S}$, and $K_{u}=2.58 \pm 0.28 \times 10^{5} \mathrm{~J} / \mathrm{m}^{3}$ (see Supplemental Material [63]), yielding $Q$ factors that are constantly smaller than 1 with an average value of $\bar{Q}=0.31 \pm 0.01$.

In summary, we see that the applied field orientation $\beta$ as well as the balance of magnetic energy contributions have a significant impact on the morphology of the remanent domain states: a full demagnetization processes may result in either aligned or randomly distributed stripe domain patterns, by simply changing either $\beta$ or $N$. In the next section, we explore the viability of 
modulating the remanent magnetic domain configuration via minor loop cycling, as suggested in prior studies [48-50].

\section{Manipulation of domain shapes}

Here, we aim towards achieving control of the remanent magnetic domain pattern structure in our $[\mathrm{Co} / \mathrm{Pt}]_{N}$ multilayers via minor loop cycling with the field applied along the OOP orientation. For this, a descending series of minor loops was applied to the sample with MFM images taken in between. Fig. 8(a) shows as (red) line normalized $M / M_{S}$ data as a function of the positive reduced field $h=\mu_{0} \mathrm{H} / \mu_{0} \mathrm{H}_{\mathrm{S}}^{\mathrm{OOP}}$ for the sample $N=20$, with $\mu_{0} \mathrm{H}_{\mathrm{S}}^{\mathrm{OOP}}$ being the OOP saturation field. The inset displays the MFM image ${ }^{8}$ of the remanent magnetic state after applying the maximum reduced field $h_{m}=1.6$. The resulting domain pattern is already quite different from the MFM image measured after the full OOP demagnetization process (Fig. 6). Indeed, the MFM measurement displayed in the inset of Fig. 8(a) is reminiscent of the elliptic bubble domains predicted by Thiele [33], consisting of randomly distributed short stripe domains. The absence of any global alignment is due to the external field possessing no significant IP component as well as its maximum strength being larger than $\mu_{0} \mathrm{H}_{\mathrm{S}}^{\mathrm{OOP}}$ in the presence of nucleation hysteresis at high field. The remanent domain pattern established after applying $h_{m}=1.6$ has been selected as the starting point for the entire minor loop investigations of each sample ${ }^{9}$. Setting $h_{m}=1$, the corresponding normalized magnetization curve plotted in Fig. 8(b) shows that the reversal still occurs as a first-order phase transition via nucleation of reverse domains. The inset shows the evolution of the remanent domain structure, in which some short stripes have split into bubble-like domains. By further decreasing the reduced field strength to $h_{m}=0.95$, as shown in Fig. 8(c), the magnetization reversal curve does not show the first order reversal behavior anymore, indicating that the magnetization mechanism here is mainly occurring via domain wall motion. By describing the curve along the ascending part, the domains magnetized opposite with respect to the external field get linearly smaller in width, before splitting in their length at sufficiently high field, thus transforming into isolated bubbles. By

\footnotetext{
${ }^{8}$ The MFM images displayed in Fig. 8 have been measured on different region of the samples, i.e., they do not correspond to the same sample area.

${ }^{9}$ Each minor magnetization loop was performed in the following way: starting from remanence ( $h=0$, after having performed a full major magnetization loop) the field strength is first increased to its maximum value $h_{m}$, then reversed down to $-h_{m}$, increased up again to $h_{m}$ and finally reduced back to 0 . At this last point the remanent MFM images were recorded.
} 
then decreasing the field back to $h=0$, the magnetic state consisting of bubbles and short stripes at $h=h_{m}$ is imprinted in the remanent domain pattern as noticeable in the inset of Fig. 8(c). This is due to the magnetostatic repulsion of the presumably high density of domains at $h_{m}$, preventing them from merging to extended stripe domains $\left[6,74,{ }^{75}\right]$.

In order to further investigate the effect of magnetic history on the remanent domain morphology, and in an attempt to better target the optimal field value $h_{m}$ maximizing the magnetic domain density, a series of MFM images at remanence were measured after applying one complete magnetization loop of amplitude $h_{m}$. The amplitude $h_{m}$ was progressively decreased in steps of 0.05 down to 0.6 for each sample. For comparison between datasets, the tip-sample distance was fixed at $40 \mathrm{~nm}$ and the color-code scale fixed at $0.5^{\circ}$ (phase shift) in all MFM measurements. The signal contrasts of different remanent state images exhibit no change, which means that the intensity of the surface stray field has also not changed within each sample. As visible in Figs. 8(d)-(f), while the $h_{m}$ values are confined in a field range corresponding to the nucleation hysteresis of the major loop, by decreasing the minor loop amplitude the shortened stripes collapse into bubbles arranging themselves into a quasi-hexagonal lattice as indicated in the top-left inset of Fig. 8(f). The existence of a metastable hexagonal bubble phase, such as the one displayed in Fig. 8, is a well-known fact for many systems in which competing interactions favor spatial inhomogeneities whose modulation can be tuned by varying for instance temperature, electric or as in this case magnetic field [38,39]. By further decreasing $h_{m}$, the minor loop extension slowly recedes from the nucleation hysteresis of the major loop. Consequently, less stripe domains reach a sufficient opposite magnetic field in order to pinch into bubble domains and thus are preserving the stripe-like geometry at remanence (which is formed from the previous bubble-rich remanent states mainly during the negative part of the minor loop, where some bubble domains grow enough to eventually merge to stripes), as shown in Figs. 8(g) and (h).

Considering the MFM images displayed in Figs. 6 and 7, we can expect that the magnetic domain period as well as its morphology is determined by the long-range and short-range competing interactions, whose ratio is varied by changing $N$. Moreover, the minimum diameter of individual bubbles as well as its ratio with respect to its domain wall width strongly depend on the balance between anisotropy and the magnetostatic energies $[33,40]$. Therefore, in order to further explore the formation of a bubble lattice at room temperature, the above described minor loop investigation has been performed as a function of the number of $\mathrm{Co} / \mathrm{Pt}$ repetitions $N$ to investigate 
the role of the thin film magnetostatic energy for the modulated phase. Fig. 9 shows the full remanent MFM characterization for the samples with $14 \leq N \leq 30$ as a function of the reduced field $h_{m}$. The samples with small $N$ were excluded from this investigation due to the absence of a first order nucleation of domains, which should prevent the formation of isolated domains at remanence.

The choice of $N$ and $h_{m}$ has a profound impact on the remanent domain pattern morphology, thus setting up a way for the effective manipulation of high-density bubble-like domain lattices. We find the field range of $0.70 \leq h_{m} \leq 0.85$ to be the optimal setting in order to stabilize remanent domain patterns which consist of bubble shaped domains, whereas for higher and lower $h_{m}$ those domains tend to interconnect into more elongated stripes. However, the samples with a low number of $\mathrm{Co} / \mathrm{Pt}$ repetitions $N$ (especially $N=14$ ) even in the optimal magnetic field range, exhibit a majority of domains adopting a stripe shape instead of the bubble shape. The observation of an increased number of elongated domains while decreasing $N$ at a fixed valued of $h_{m}$ is mainly caused by the thickness driven reorientation transition towards an easy-plane behavior, which was observed when analyzing the VSM data. Also, for small $N$, slight misalignments of the magnetic field direction with the OOP orientation introduces, as corroborated in Fig. 6, a preferential IP direction along which the domain patterns align. In the case of $\mathrm{Co} / \mathrm{Pt}$ multilayers with higher $N$ values, decreasing $h_{m}$ below the nucleation hysteretic field range causes the number of stripe-like domains to increase substantially as can be seen in Fig. 9 for $h_{m}<0.7$ and $20 \leq N \leq 30$. Indeed, by performing a full demagnetization process, i.e. $h_{m}=0$, all isolated magnetic domains merge into very-long connected domains that form a maze-like pattern, as displayed in Fig. 6.

For all measurements in Fig. 9, we defined the remanent domain density $\rho$ as the number of domains per $100 \mu \mathrm{m}^{2}$ area. Calculated values of $\rho$ are indicated within the inset of the MFM images shown in Fig. 9, where $\rho$ values are seen to range in about an order of magnitude, between 200 and more than 2000. In addition, the specific dependence of $\rho$ on $h_{m}$ is displayed in Fig. 10 for samples with $N \geq 14$. For all samples shown here, the density of domains for $h_{m}<0.5$ is relatively small; however, by further increasing $h_{m}, \rho$ increases significantly up to its maximum value $\rho_{\max }$. Beyond this point, the density decreases again and reaches values close to zero at and above saturation for low $N \leq 16$ [Figs. 10(a) - (b)], but keeps high offset values for $N \geq 18$ [Figs. 10(c) (i)]. Indeed, as the inset MFM images in Figs. 10(a) - (i) show for $h_{m}=1.6$, the remanent domain pattern consists of very long stripe domains for $N=14$ and 16 showing a zigzag structure that might be induced due to magnetic tensile strain originating from the slight misalignment of the magnetic 
field direction with respect to the surface normal [11]. Nevertheless, for $N \geq 18$, the remanent domain patterns for $h_{m}=1.6$ are characterized by a mixed state made of short stripes and bubbles, whose density increases considerably with respect to the low- $N$ cases. This change of the remanent domain pattern after applying $h_{m}>1$ can be ascribed to the IP reorientation transition occurring for samples with $N \leq 18$. A considerable in-plane component of the magnetization may be responsible for the increased length of domains and consequently for the change in their morphology.

The optimal reduced field stabilizing the highest density of domains at remanence was determined by fitting the experimental data in Figs. 10 (a)-(i) to the bi-Gaussian function:

$$
\begin{array}{ll}
\rho=\rho_{0_{1}}+A_{1} e^{-\frac{\left(h-h_{\max }\right)^{2}}{2 w_{1}^{2}}} & h \leq h_{\max } \\
\rho=\rho_{0_{2}}+A_{2} e^{-\frac{\left(h-h_{\max }\right)^{2}}{2 w_{2}^{2}}} & h_{\max } \leq h
\end{array}
$$

with $h_{\max }$ being the reduced field at which the highest density is found. Hereby, the offsets $\rho_{0_{i}}$, the peak widths $w_{i}$, the scaling factor $A_{i}(\mathrm{i}=1,2)$ and $h_{\max }$ were utilized as fit parameters, with the constrain $\rho_{0_{1}}+A_{1}=\rho_{0_{2}}+A_{2}$ (continuity at the peak). Figs. 10 (a)-(i) shows the fitting results as red solid lines in direct comparison to the experimental data. In each case, an excellent agreement has been found between the experimental data and the least-squares fit according to Eq. (1). The extracted values for $h_{\max }$ together with the associated errors estimated from the fits are shown in Fig. 10(j) as a function of $N$. Despite small variations in between the extracted optimal reduced fields for the samples, all $h_{\max }$ values are consistent within their average value $\bar{h}_{\max }=0.80 \pm 0.07$ that has been determined from the experiments and in agreement with earlier estimates for $N=50$ [49].

With the purpose of summarizing our experimental results in a global picture, we have used the maximum density values $\rho_{\max }$ extracted from the MFM images in Fig. 9 in order to build a $N$ $h_{m}$ remanent state phase diagram, which illustrates the measured magnetic domain densities at remanence. In the density map, which is displayed as a color-coded plot in Fig. 11(a), the maximum domain density for any investigated sample is reached in the region $0.70 \leq h_{m} \leq 0.85$. Moreover, it can be clearly seen that the maximum domain density is observed for $18 \leq N \leq 20$. In fact, the maximum value $\rho_{\max }$ is observed for $\left(N, h_{m}\right)=(18,0.80)$, where we have obtained a value of 2274 \pm 160 domains per $100 \mu \mathrm{m}^{2}$. In addition, there are two predominant magnetic configurations that consist of stripes and bubbles. The former configuration changes its morphology depending on the 
number of $\mathrm{Co} / \mathrm{Pt}$ repetitions, because of the change in the energy landscape. For both high $N$ and $h_{m}$, the samples show at remanence short stripe domains whereas by substantially decreasing the reduced field the domains increase their length forming labyrinth domains. On the other hand, for low $N$ values, canted short stripe domains have been measured for a large range of $h_{m}$ strength. Importantly, the region of the map with the highest number of domains corresponds in majority to bubbles. In fact, our phase diagram in Fig. 11(a) suggests that the morphological stripe-bubble magnetic transition is accompanied by a significant enhancement in the domain density. In Fig. 11(b), the maximum density $\rho_{\max }$ is plotted as a function of $N$. From here, one can observe that when the number of $\mathrm{Co} / \mathrm{Pt}$ repetitions approaches $18 \leq N \leq 20$, the domain density at remanence maximizes. By combining our experimental data with that in Ref. 49, a more complete $t_{C o}-N$ color-coded domain density map can be constructed, which is shown as a color coded map in Fig. 11(c). At the fixed number of $\mathrm{Co} / \mathrm{Pt}$ repetitions $N=50$, a strong increase of the remanent domain density at a thickness of $t_{\mathrm{Co}} \sim 3 \mathrm{~nm}$ corresponding to 1200 domains per $100 \mu \mathrm{m}^{2}$ was found [49]. However, one can additionally see from the outcome of our experiments that via decreasing the number of $\mathrm{Co} / \mathrm{Pt}$ repetitions, the density of domains can be further increased up to the value of $\rho=$ $2274 \pm 160$ domains per $100 \mu \mathrm{m}^{2}$ for $N=18$ [see Fig. 11(b)]. As previously pointed out, the domain density decreases again for $N<18$, as a result of the gradual IP reorientation transition of magnetization.

Finally, we have also estimated the fundamental length scales of the bubble-like domain lattices stabilized in our $[\mathrm{Co} / \mathrm{Pt}]_{N}$ multilayers. Fig. 11(d) displays the characteristic nearest neighbor distance $D$ between bubbles as a function of the average bubble radius $r$, the definition of both being schematically defined in the inset of the figure. We see that by reducing the number of $\mathrm{Co} / \mathrm{Pt}$ repetitions, the average bubble radius decreases monotonically, in good agreement with previous findings on garnet systems [76]. This property allows the system to arrange more and more bubbles along a given line by reducing their distance $D$. Also the estimate of the bubble size at $N=50$ (radius of about $75 \mathrm{~nm}$ [49]) agrees well with the trend shown in Fig.11 (d). Moreover the sizes of bubble domains found in this work are close to values reported for skyrmions, which were measured in samples having similar magnetization curves [77]. However, despite the strong spinorbit coupling at the $\mathrm{Co} / \mathrm{Pt}$ interfaces, there should not be any topological protection for those spins textures in our samples, since our multilayer systems should lack of any broken inversion symmetry. Therefore, the observed magnetic bubbles shown in Fig. 9 should have collinear 
alignment of spins excluding their domain walls, which may be further investigated by TEM in order to evaluate the distribution of their chiralities, in particular the ratio of clockwise versus counter clockwise oriented bubble domain walls. Indeed, we expect this ratio to be roughly equal to $1 / 2$, as well as some achiral bubbles to be present, as we did not purposely introduce any inversion symmetry breaking features. However in some studies [78], even in Co/Pt multilayers, some degree of DMI was observed due to the fact that Co layers do not grow on Pt exactly the same as vice versa, which may still cause some relevant inversion symmetry breaking.

\section{IV.Conclusions}

In this paper, we have successfully fabricated $[\mathrm{Co}(3.0 \mathrm{~nm}) / \operatorname{Pt}(0.6 \mathrm{~nm})]_{N}$ multilayer films with a magnetic easy axis perpendicular to the film plane and narrow $c$-axis dispersion. The room temperature magnetometry measurements for the sample with $N=30$ reveal the presence of two very different magnetization reversal processes depending on the applied field angle $\beta$, namely an instability-driven process leading to the generation of stripe domains for $\beta$ values near IP field orientation and a nucleation domain process near OOP field orientation that is hysteretic in nature. Our $N$-dependent study shows a gradual shrinking of the nucleation regime, so that at sufficiently low $N$ only the instability-driven second-order phase transition occurs for all angles $\beta$. The disappearance of the nucleation regime is driven by the strong thickness dependent balance between magnetic anisotropy and magnetostatic energies and occurs before the effective OOP anisotropy energy becomes too weak to support a stripe domain state altogether, i.e. it occurs while the stripe domain instability is still dominating the magnetization reversal process. By further reducing the number of $\mathrm{Co} / \mathrm{Pt}$ repetitions below $N<10$, we observe a characteristic easy-plane magnetization behavior. Furthermore, the evolution of the samples remanent magnetic domain structures with magnetic field history was explored by magnetic force microscopy. It was found that the remanent domain configuration can be gradually transformed from stripes to bubbles after applying different magnetic field sequences along the out-of-plane direction. This magnetic transition was identified by mapping out the density of the domains as a function of reduced field $h_{m}$ and $N$. The resulting MFM data shows the magnetic transition with the occurrence of a region of a high-density bubble domain remanent state for $0.70 \leq h_{m} \leq 0.85$ and $18 \leq N \leq 20$. The domain density in this region is significantly enhanced to $\sim 2300$ domains $/ 100 \mu \mathrm{m}^{2}$. The present work provides therefore a feasible approach for manipulating magnetic domains in $\mathrm{Co} / \mathrm{Pt}$ multilayer film 
by finely adjusting the magnitude of the previously applied external magnetic field strength and the energetic landscape of the system. Furthermore, it is shown that the evolution of the domain structure can be controlled, which provides the possibility of manipulating magnetic domains with the applied field, for possible applications in spin-electronic or logic devices. Lastly, the demonstrated feasibility of tuning $r$ and $D$ of bubble-shaped domains by changing the number of $\mathrm{Co} / \mathrm{Pt}$ repetitions, while at the same time keeping their morphology constant, may resemble a lithographic patterning process for creating two-dimensional dot or anti-dot lattices of varying dimensions. As for the samples presented here, the periodicity of the magnetic modulation as well as the modulation itself can be tuned and controlled by the strength of the external applied field and its orientation. This novel approach of all-magnetic patterning can prove useful in order to fabricate novel devices such as magnonic crystals [79]. Therefore, the thick $\mathrm{Co} / \mathrm{Pt}$ multilayer systems presented here could constitute a relevant building block to be employed in magnonic devices in order to achieve specific and field-reconfigurable spin wave propagation and dispersion relations. Given the generality of our observations we expect that our findings might extend to many different ferromagnetic materials that exhibit a strong competition between long- and shortrange interactions. As a final remark, one could envision the multilayer system investigated in this work to become suitable for skyrmion physics. By purposely introducing significant DMI via controlled inversion symmetry breaking, for instance by replacing the $[\mathrm{Pt} / \mathrm{Co}]_{N}$ bilayer by $[\mathrm{Pt} / \mathrm{Co} / \mathrm{Ir}]_{N},[\mathrm{Pt} / \mathrm{Co} / \mathrm{Ta}]_{N}$ or $[\mathrm{Pt} / \mathrm{Co} / \mathrm{Fe} / \mathrm{Ir}]_{N}[80,81]$, and by reducing the thickness of the individual Co layers while keeping constant $N=20$ (which corresponds to the highest bubble domain density at remanence in our work), one should be able to find an optimal set of material parameters able to stabilize a remanent bubble state with one specific chirality.

\section{ACKNOWLEDGMENTS}

L. F. is thankful to Andreas Berger, Sven Stienen and Jürgen Lindner for fruitful discussions as well as to Kay Potzger for the support with experimental facilities. J. A. A. acknowledges the Grant Agency of the Czech Republic (grant No. 16-23940Y). Part of the work was carried out with the support of CEITEC Nano Research Infrastructure (ID LM2015041, MEYS CR, 2016-2019), CEITEC Brno University of Technology. This work has received funding from the European Union's Horizon 2020 research and innovation program under the Marie Skłodowska-Curie and it is co-financed by the South Moravian Region under grant agreement No. 665860. 


\section{FIGURE 1}
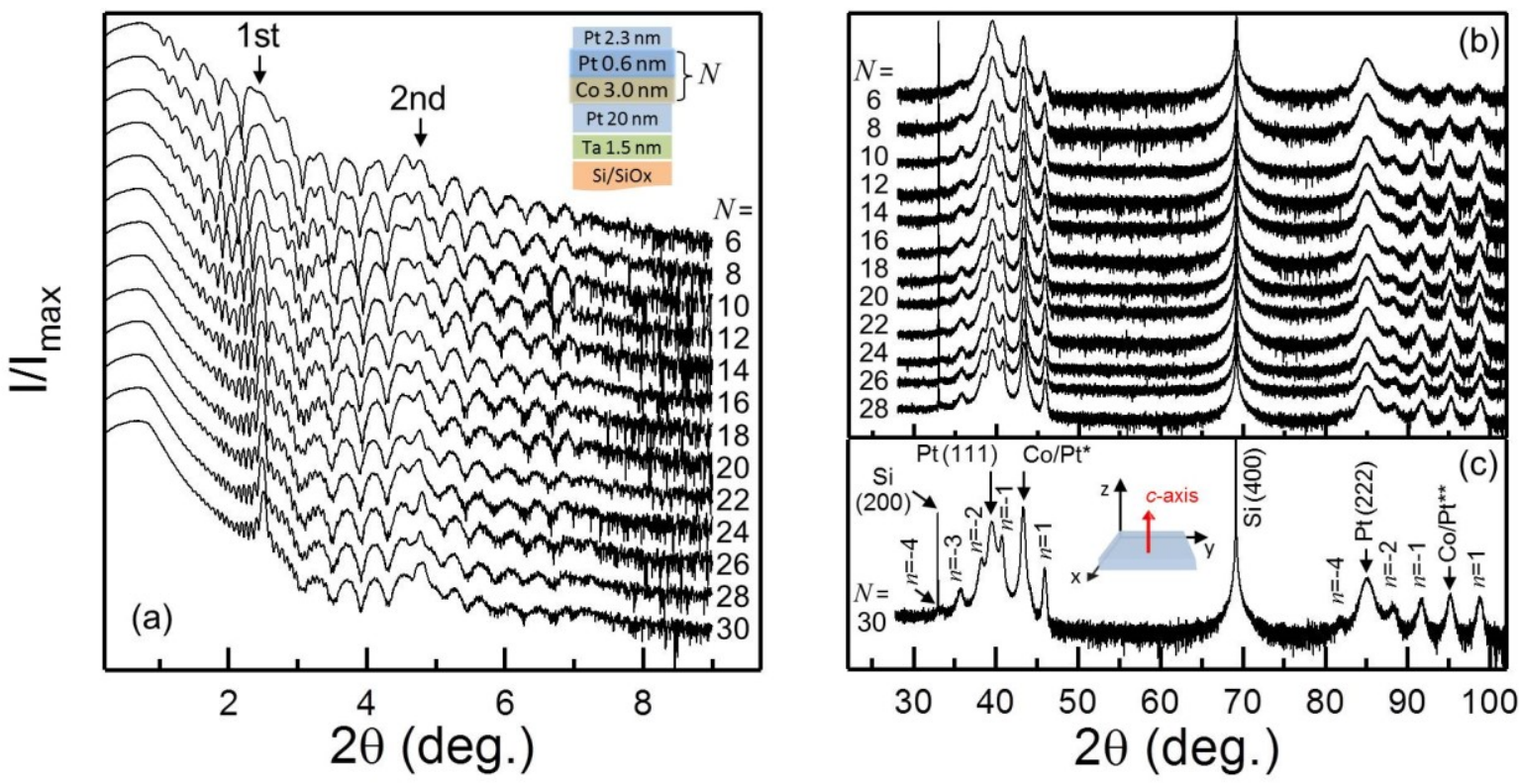

Fig. 1 (color online) (a) XRR $\omega / 2 \theta$ scans for different samples with Co/Pt repetitions $6 \leq N \leq 30$. The inset shows a schematic of the layer growth sequence for the sample type explored in this study. (b) XRD $\theta / 2 \theta$ measurements of the same samples as in (a): each scan has been normalized to the intensity of its $\mathrm{Si}(400)$ substrate peak. (c) XRD $\theta / 2 \theta$ scan for the sample $N=30$, which shows only $\mathrm{Pt}$ (111), $\mathrm{Co} / \mathrm{Pt}^{*}$ and its satellite peaks $n$, as well as the corresponding second order diffraction peaks, in addition to the Si (200) and Si (400) substrate signals. The indexing in (c) serves as reference for the scans displayed in (b). The Co/Pt* label refers to $\mathrm{Co}(0002) / \mathrm{Pt}(111)$ and $\mathrm{Co} / \mathrm{Pt}^{* *}$ to $\mathrm{Co}(0004) / \mathrm{Pt}(222)$. 


\section{FIGURE 2}
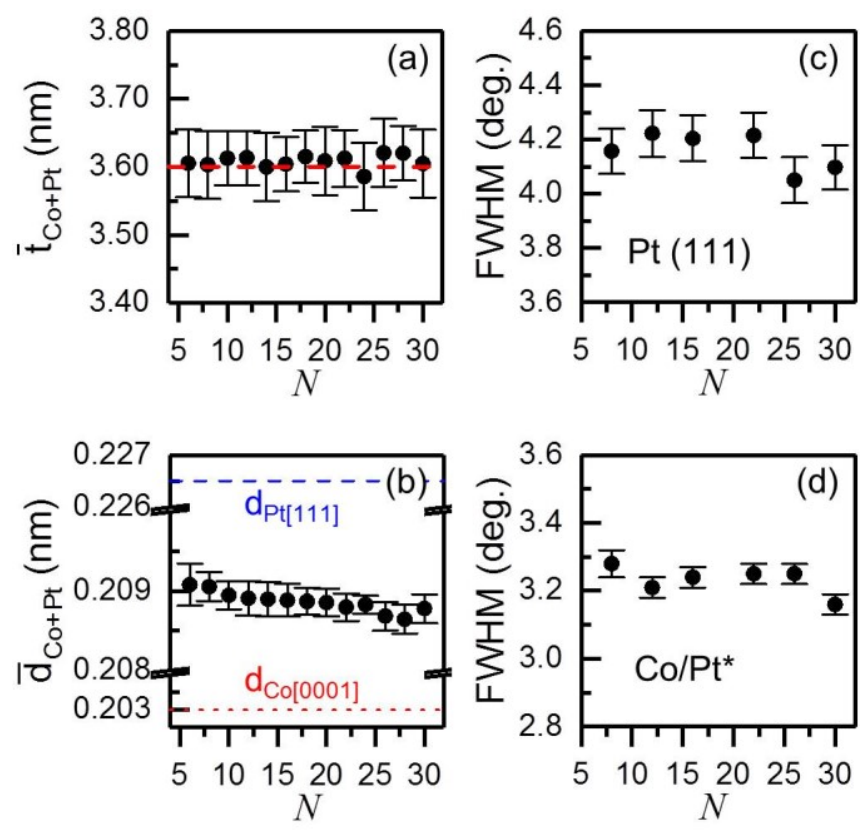

Fig. 2 (color online) (a) Average total thickness of the single Co/Pt bilayer $\bar{t}_{\mathrm{Co}+\mathrm{Pt}} \mathrm{vs} . N$ as obtained from XRR measurements. The (red) dashed line indicates the nominal $t_{\mathrm{Co}+\mathrm{Pt}}=3.6 \mathrm{~nm}$. (b) Average out-of-plane interplanar distance $\bar{d}_{\mathrm{Co}+\mathrm{Pt}}$, of the Co/Pt heterostructure vs. $N$. The (blue) dashed line and the (red) dotted line indicate the Pt (111) and Co (0002) interplanar distances, respectively. (c) FWHM of the rocking curve measurements performed at the $\mathrm{Pt}(111)$ diffraction angle as a function of $N$. (d) FWHM of the rocking curve measurements performed at the $\mathrm{Co} / \mathrm{Pt}^{*}$ heterostructure diffraction peak as a function of $N$. The Co/Pt* label refers to Co (0002) / Pt (111). 


\section{FIGURE 3}
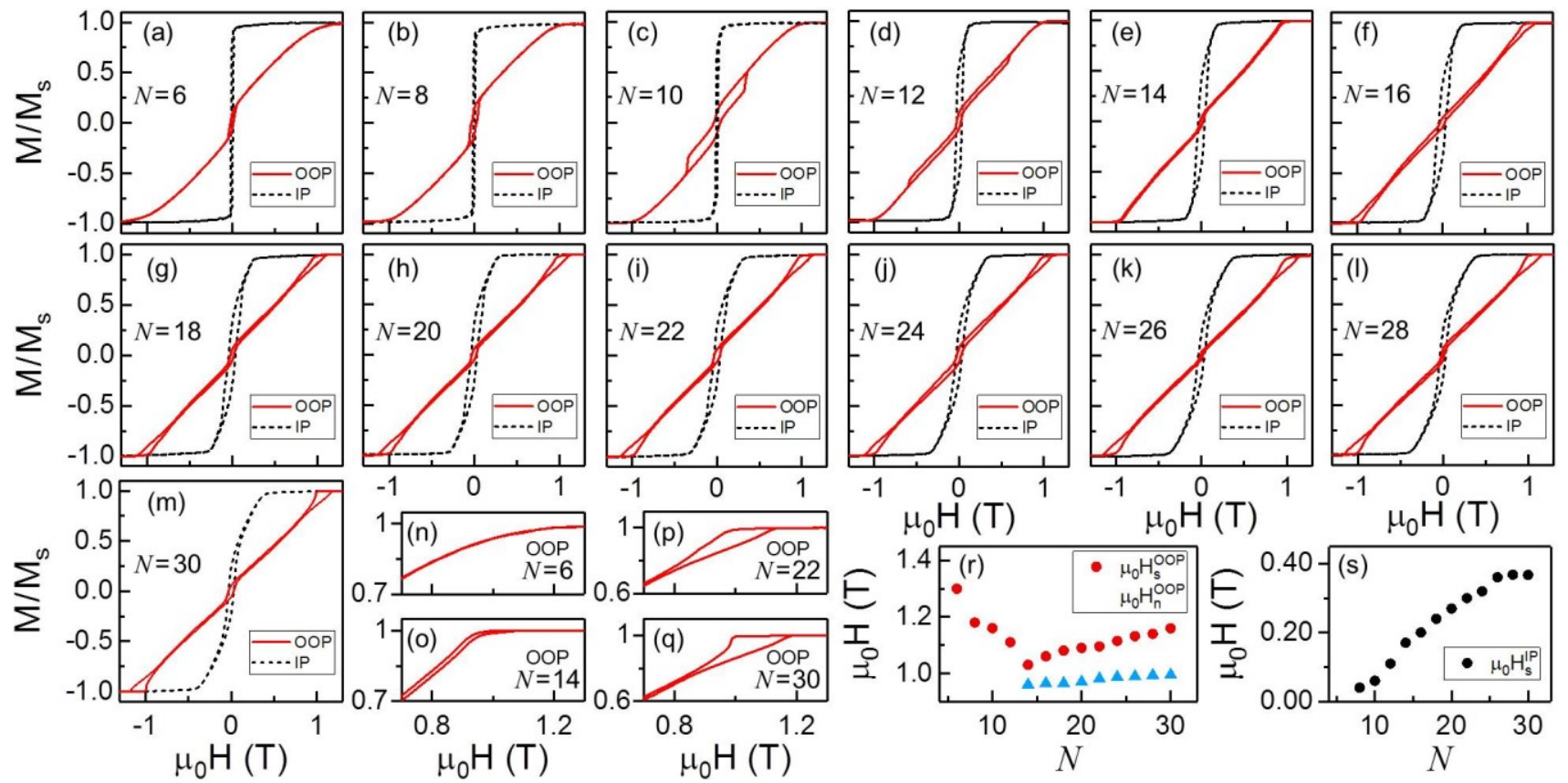

Fig. 3 (color online) (a)-(m) VSM room temperature magnetization reversal curve measurements with the applied field along the IP (black) and OOP (red) directions for the entire set of $[\mathrm{Co} / \mathrm{Pt}]_{N}$ samples. The data are normalized to its maximum value $M_{S}$ in each case. (n)-(q) Zoomed-in view of the OOP magnetization reversal curve for four selected samples with (n) $N=6$, (o) 14, (p) 22 and (q) 30. (r)-(s) $N$-dependence of the OOP-saturation magnetic field $\mu_{0} \mathrm{H}_{\mathrm{S}}^{\mathrm{OOP}}$ (red circles), the nucleation magnetic field $\mu_{0} \mathrm{H}_{\mathrm{n}}^{\mathrm{OOP}}$ (light blue triangles) and IP saturation magnetic field $\mu_{0} \mathrm{H}_{\mathrm{s}}^{\mathrm{IP}}$. 


\section{FIGURE 4}

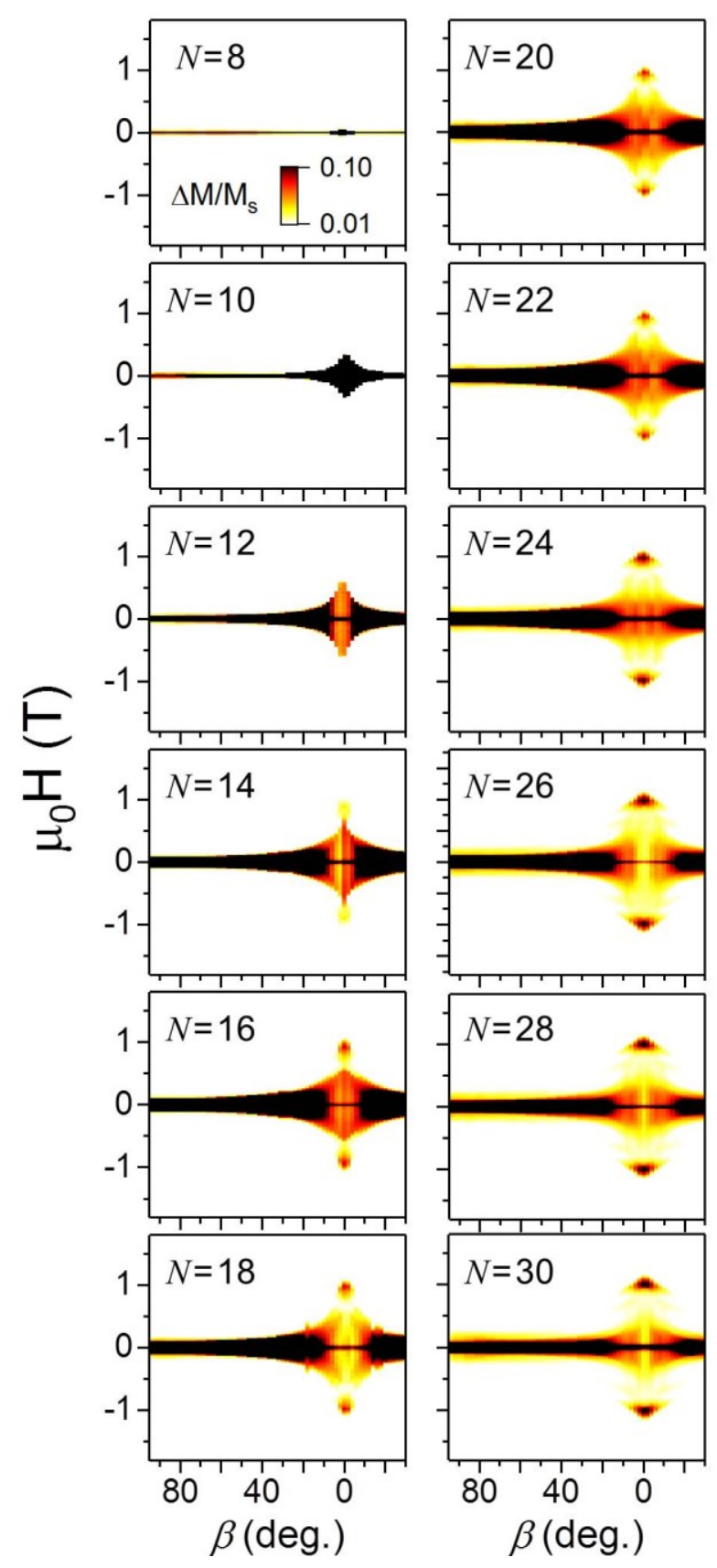

Fig. 4 (color online) $\Delta M / M_{S}$ (color-coded) maps as a function of field angle $\beta$ and strength $\mu_{0} H$ measured for different samples with $8 \leq N \leq 30$ as indicated in each map. The scale (color code) is defined in the figure. $\beta=0^{\circ}$ corresponds to $H$ applied along the surface normal (OOP), $\beta=90^{\circ}$ refers to IP field orientation. The $\Delta \mathrm{M} / \mathrm{M}_{S}$ values are defined as the difference between the ascending and descending branches of the magnetization curves. 


\section{FIGURE 5}
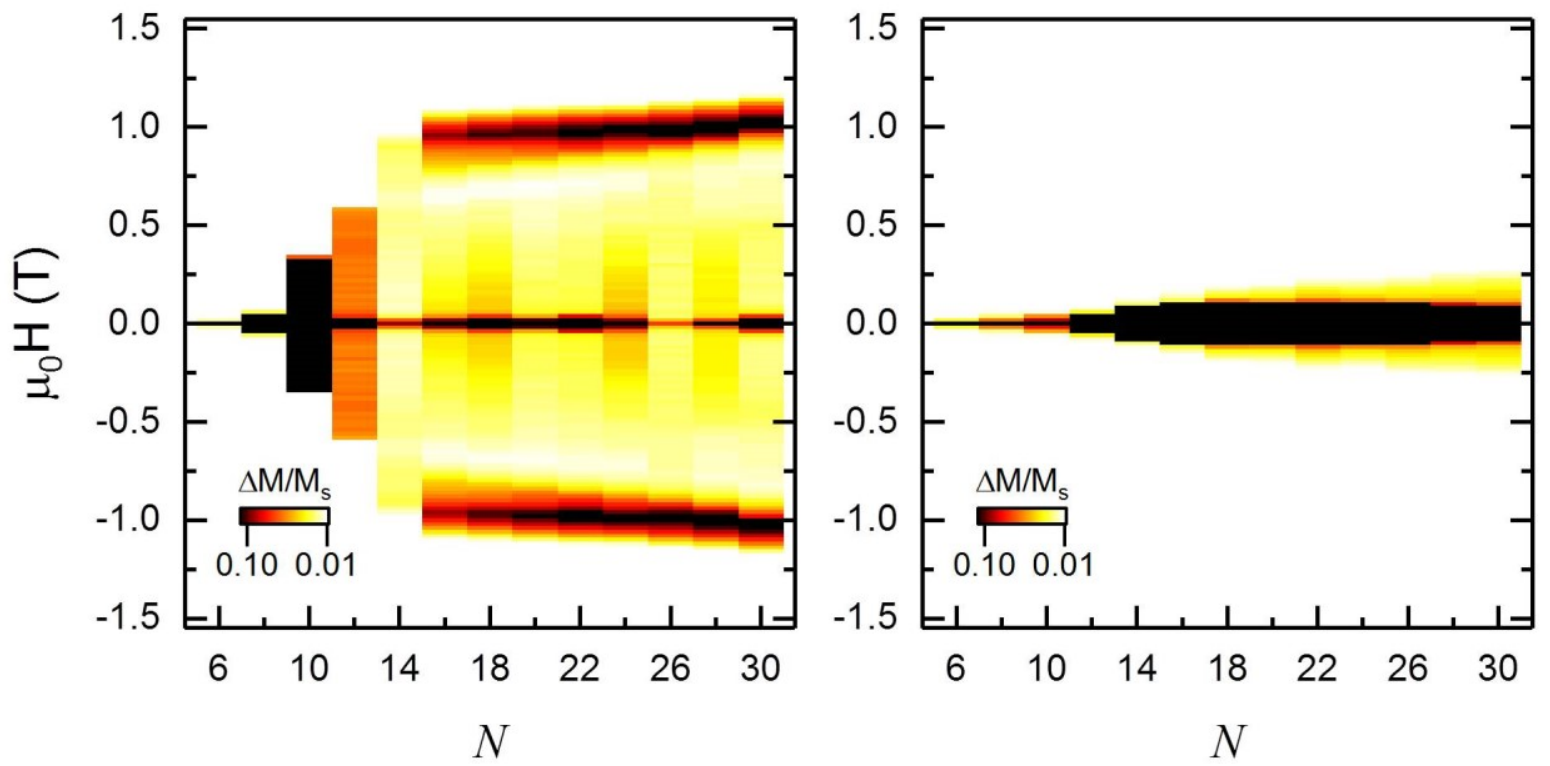

Fig. 5 (color online) $\Delta M / M_{S}$ (color-coded) maps as a function of field strength $\mu_{0} \mathrm{H}$ and number of $\mathrm{Co} / \mathrm{Pt}$ bilayers $N$ for applied field angle values (a) $\beta=0^{\circ}$ and (b) $90^{\circ}$. The scale (color code) is defined in the figure. 


\section{FIGURE 6}
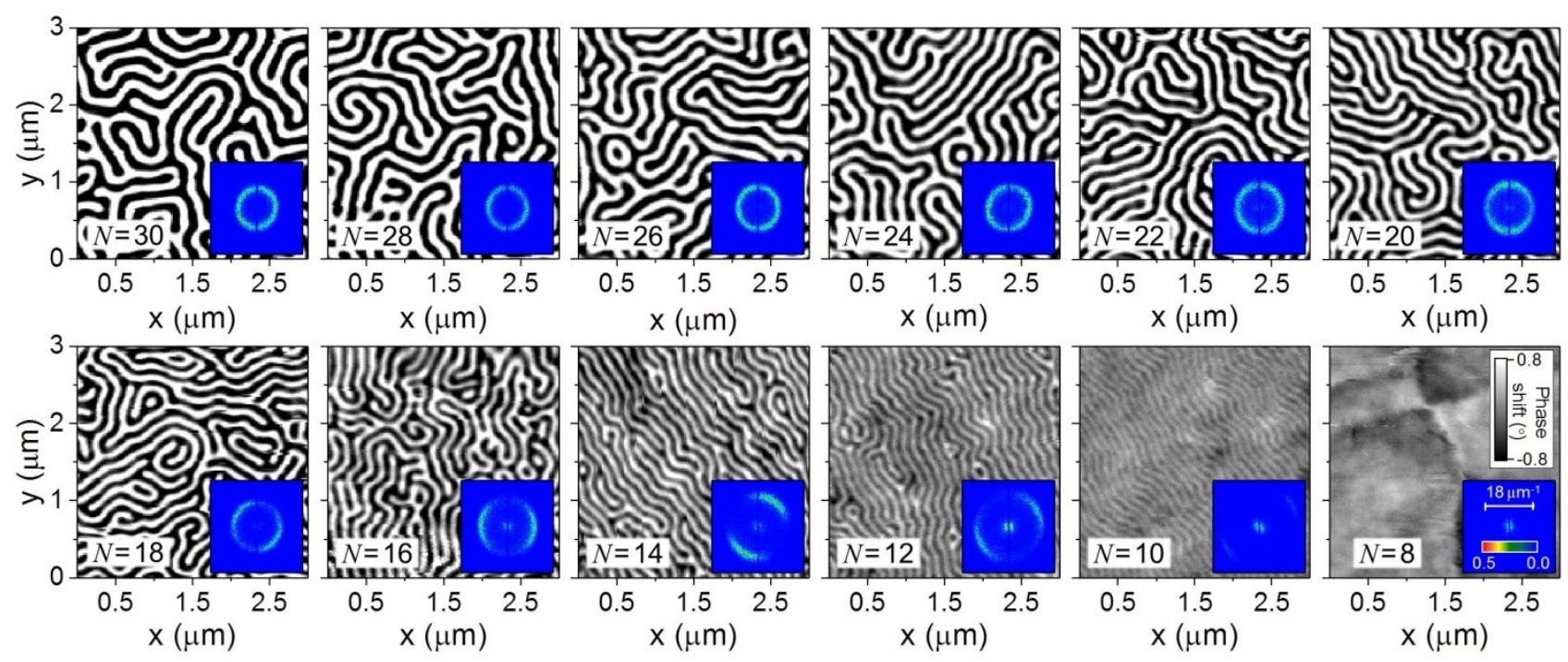

Fig. 6 (color online) Remanent MFM images recorded after the OOP demagnetization process as a function of $N$. The same color scale (gray code) is used for all the images, as defined in the figure for the sample $N=8$. The insets display the normalized to the maximum two-dimensional fast Fourier transform of the MFM image shown in the same figure. The same scale (color code) is used for all the 2D-FFT images, as defined in the inset of the figure for the sample $N=8$. 


\section{FIGURE 7}
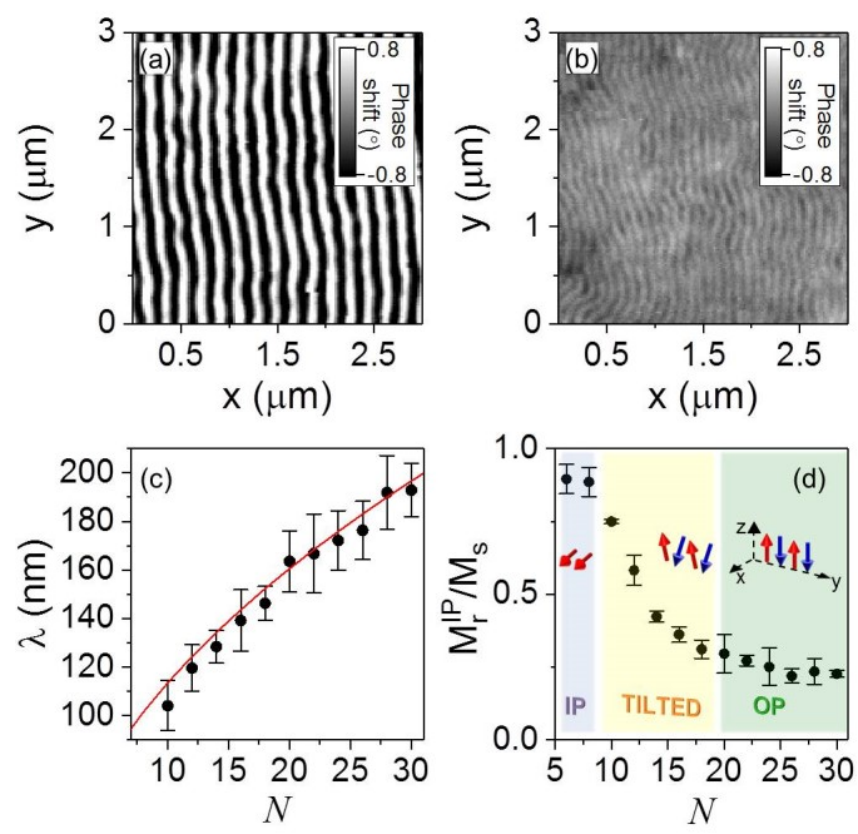

Fig. 7 (color online) Remanent MFM images measured after the IP demagnetization process for the samples with (a) $N=30$ and (b) $N=10$. The scale (gray code) is defined in each figure. (c) shows the magnetic domain periodicity $\lambda$ as a function of $N$ (black squares) together with the calculated stripe domain period (red solid line) based on analytical expression developed in $[6,31,69,72]$ (see Supplemental Material [63]). (d) displays the $N$-dependence of the in-plane remanence ratio $M_{r}^{I P} / M_{S}$ (obtained from the magnetometry data of Fig.3) together with schematics indicating the remanent magnetic state in the three different regions. 


\section{FIGURE 8}
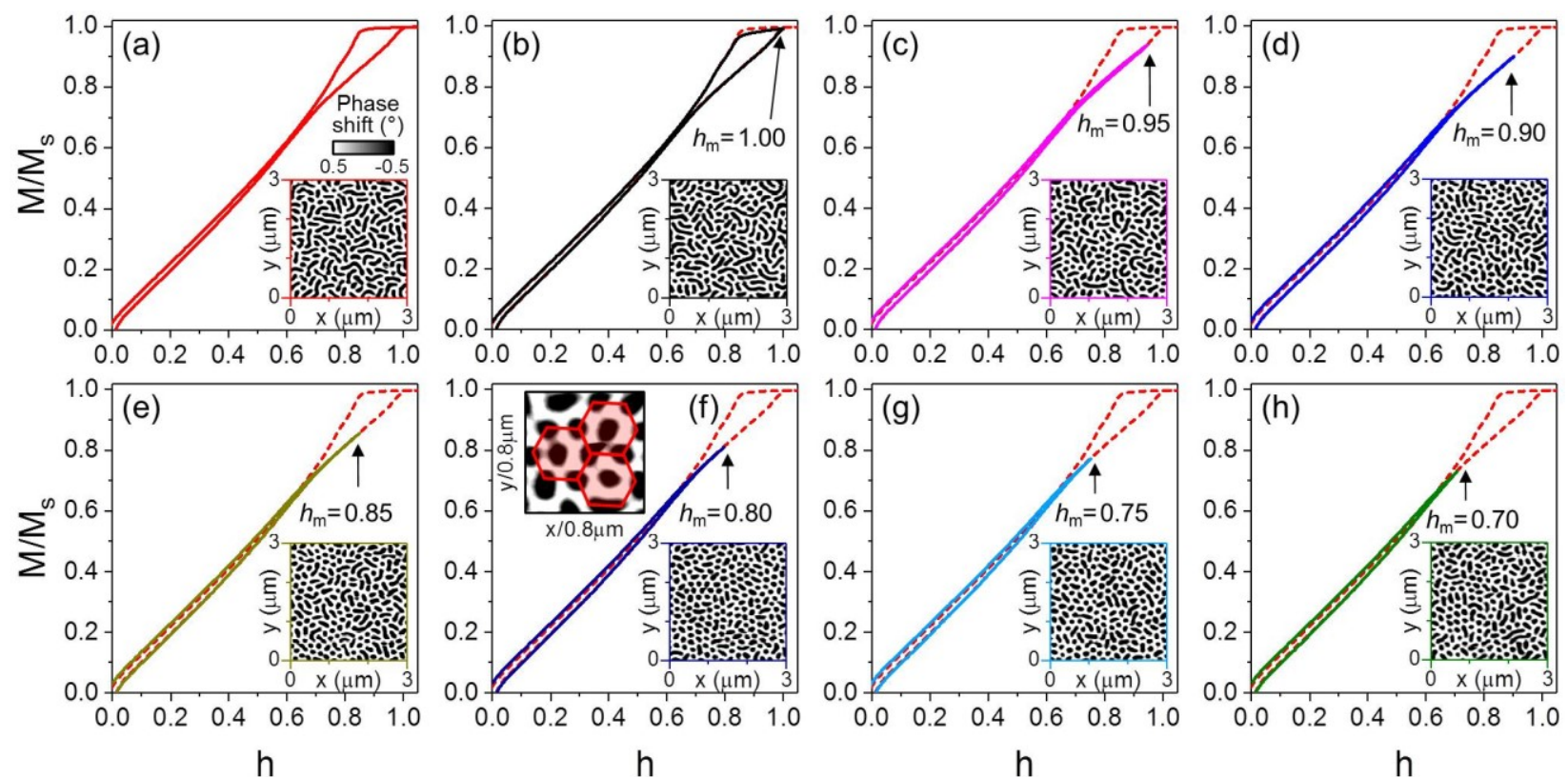

Fig. 8 (color online) VSM room temperature magnetization reversal curve measurements along the OOP field direction for the sample with $N=20$. Hereby, the magnetization is normalized to the maximum value $M_{\mathrm{S}}$, whereas the magnetic field values are divided by $\mu_{0} \mathrm{H}_{\mathrm{S}}^{\mathrm{OOP}}$. In (a) the full VSM major loop is displayed with the inset showing the remanent magnetic domain configuration after the field $h_{m}=1.6 \times \mu_{0} \mathrm{H}_{\mathrm{S}}^{\mathrm{OOP}}$ well above saturation was applied. (b)-(h) show VSM minor loops where the externally applied magnetic field does not exceed $h_{m}=\gamma \times \mu_{0} \mathrm{H}_{\mathrm{S}}^{\mathrm{OOP}}$, with $\gamma=[1.00$ (black, b), 0.95 (magenta, c), 0.90 (blue, d), 0.85 (gold, e), 0.80 (blue, f), 0.75 (royal, g), 0.70 (green, h)]. Each inset shows the remanent magnetic domain configuration after the corresponding $h_{m}$ was applied. Moreover, the complete magnetization reversal curve displayed in (a) is also plotted as dashed (red) line in (b-h) for reference in the background. The left inset in (f) shows a zoomed area of the MFM images depicted on the right-hand side, with a hexagonal-like lattice illustrated schematically. 


\section{FIGURE 9}

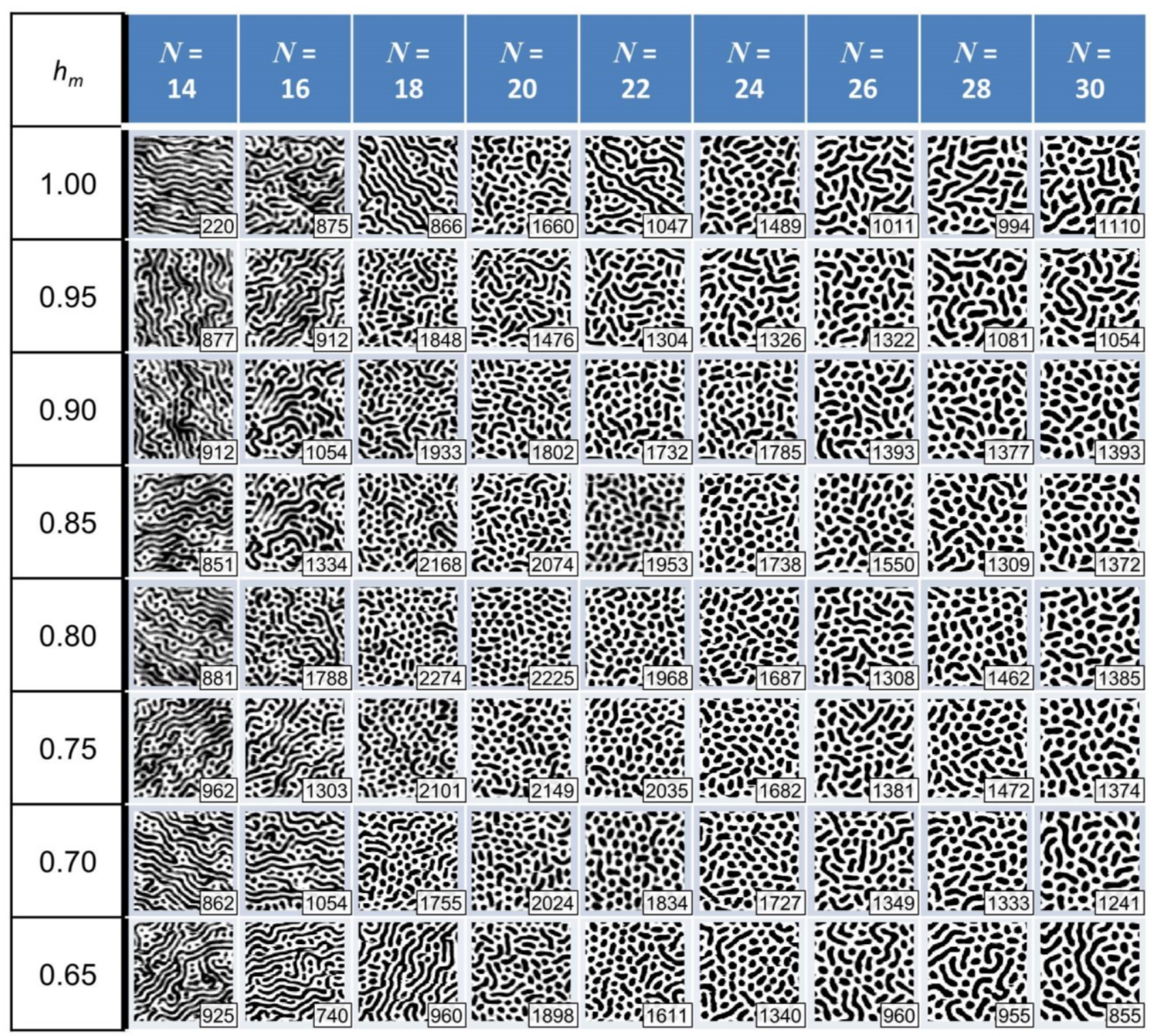

Fig. 9 (color online) Remanent MFM images measured as a function of $N$ and the reduced magnetic field strength $h_{m}$. Each MFM image covers a $2 \times 2 \mu \mathrm{m}^{2}$ area. The scale (grey code) is the same as in Figs. 6 and 7. The number at the corner of each MFM image refers to the magnetic domain density $\rho$ (number of domains per 100 microns square) at remanence (see main text). 


\section{FIGURE 10}
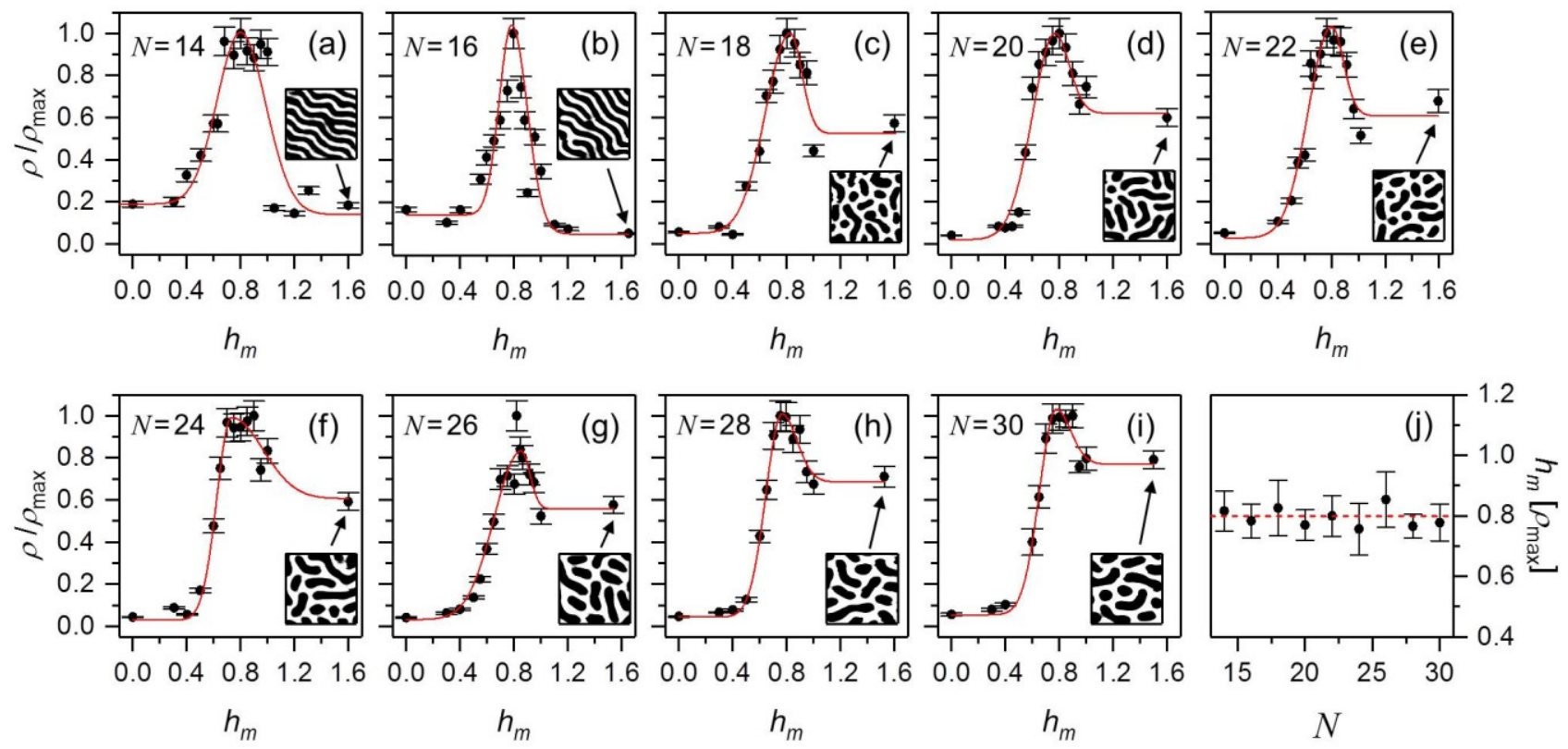

Fig. 10 (color online) (a)-(i) Normalized remanent magnetic domain density $\rho$ as a function of $h_{m}$ and for $14 \leq N \leq 30$. The (red) solid lines represent the least-squares fits to Eq. (1) for each of the sample data sets. The insets in each figure display $3 \times 3 \mu \mathrm{m}^{2}$ MFM images, which were measured after applying $h_{m}=1.6$. (j) reduced magnetic field corresponding to the maximum remanent magnetic domain density $h_{m}\left[\rho_{\max }\right]$ as a function of $N$. The (red) dashed line indicates the weighted average. 


\section{FIGURE 11}
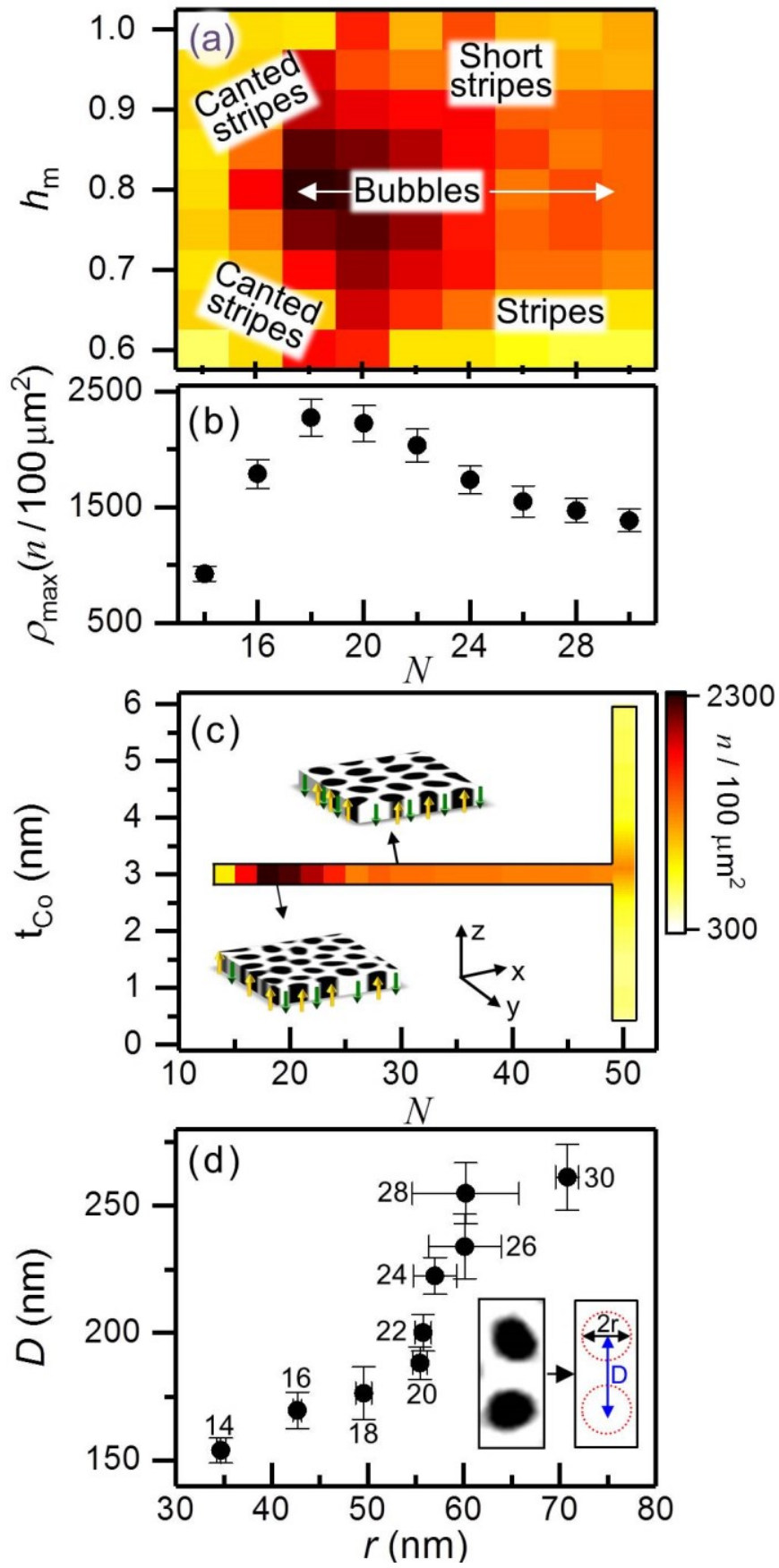
Fig. 11 (color online) (a) Color-coded map of the magnetic domain density at remanence as a function of $h_{m}$ and $N$. The scale (color code) is defined in (c). (b) Maximum domain density $\rho_{\max }$ as a function of $N$. (c) Color-coded map of the maximum magnetic domain density at remanence as a function of $N$ and the thickness of the individual cobalt layers $t_{C o}$. The data corresponding to $N=50$ is adapted from [49], whereas the data for $\left(31 \leq N \leq 50, t_{\text {Co }}=3.0 \mathrm{~nm}\right)$ are a linear interpolation based on both studies. The area investigated by this study and Ref. 49 is delimited by thick (black) borders. The insets illustrate two areas covered by only bubble-like domains for the samples $N=30$ (inset I) and $N=20$ (inset II), with the arrows referring to the local orientation of the magnetization either up (yellow arrow and black color) or down (green arrow and white color). The z-dimension has been artificially created while assuming the absence of depth dependence domain shape modulation. (d) Average nearest neighbor distance between bubbles $D$, plotted as a function of bubble radius $r$. The schematic inset shows the definition of the quantities plotted in (d). The number accompanying the data-points in the plot is the repetition number $N$ of $\mathrm{Co} / \mathrm{Pt}$ multilayer system. 


\section{References}

[1] C. Kittel, Rev. Mod. Phys. 21, 541-583 (1949); C. Kittel and J. K. Galt, Solid State Phys.

3, 437-565 (1956).

[2] A. Hubert and R. Schäfer, Magnetic Domains (Springer-Verlag Berlin Heidelberg, 1998).

[3] R. Allenspach, M. Stampanoni, and A. Bischof, Phys. Rev. Lett. 65, 3344 (1990).

[4] R. Allenspach J. Magn. Magn. Mat. 129, 160-185 (1994).

[5] D. M. Donnet, K. M. Krishnan, and A. Yajima, J. Phys. D 28, 1942 (1996).

[6] M. Hehn, S. Padovani, K. Ounadjela, and J. P. Bucher, Phys. Rev. B 54, 3428 (1996).

[7] M. Hehn, K. Cherifi-Khodjaoui, K. Ounadjela, J. P. Bucher, and J. Arabski, J. Magn. Magn. Mat. 165, 520-523 (1997).

[8] M. Hehn, K. Ounadjela, R. Ferrè, W. Grange, and F. Rousseaux, Appl. Phys. Lett. 71, 2833 (1997).

[9] V. Gehanno, Y. Samson, A. Marty, B. Gilles, and A. Chamberod, J. Magn. Magn. Mater. 172, 26-40 (1997).

[10] J. U. Thiele, L. Folks, M. F. Toney, and D. K. Weller, J. Appl. Phys. 84, 5686 (1998).

[11] M. Demand, S. Padovani, M. Hehn, K. Ounadjela, and J. P. Bucher, J. Magn. Magn. Mat. 247, 147-152 (2002).

[12] A. K. Suszka, A. Etxebarria, O. Idigoras, D. Cortés-Ortuño, P. Landeros, and A. Berger, Appl. Phys. Lett. 105, 222402 (2014).

[13] L. Fallarino, O. Hovorka, and A. Berger, Phys. Rev. B 94, 064408 (2016).

[14] O. Hellwig, T. L. Kirk, J. B. Kortright, A. Berger, and E. E. Fullerton, Nat. Mat. 2, 112 (2003).

[15] O. Hellwig, A. Berger, and E. E. Fullerton, Phys. Rev. Lett. 91, 197203 (2003).

[16] J. E. Davies, O. Hellwig, E. E. Fullerton, G. Denbeaux, J. B. Kortright, and K. Liu, Phys. Rev. B 70, 224434 (2004).

[17] O. Hellwig, A. Berger, J. B. Kortright, and E. E. Fullerton, J. Magn. Magn. Mat. 319, 13-55 (2004).

[18] R. Sbiaa, Z. Bilin, M. Ranjbar, H. K. Tan, S. J. Wong, S. N. Piramanayagam, and T. C. Chong, J. Appl. Phys. 107, 103901 (2010).

[19] L. Belliard, J. Miltat, V. Kottler, V. Matht, C. Chappert, and T. Valet, J. Appl. Phys. 81, 5315 (1997). 
[20] M. Labrune and A. Thiaville, Eur. Phys. J. B 23, 17-28 (2001).

[21] C. Bran, A. B. Butenko, N. S. Kiselev, U. Wolff, L. Schultz, O. Hellwig, U. K. Rößler, A. N. Bogdanov, and V. Neu, Phys. Rev. B 79, 024430 (2009).

[22] J. L. Archer, L. Tocci, P. K. George, and T. T. Chen, IEEE Trans. Magnetics 8, 695-700 (1972).

[23] A. H. Eschenfelder, Magnetic Bubble Technology Vol. 14 (Springer Berlin, 1980).

[24] T. H. O’Dell, Rep. Prog. Phys. 49, 589 (1986).

[25] K. Kudo, M. Suzuki, K. Kojima, T. Yasue, N. Akutsu, W. A. Diño, H. Kasai, E. Bauer, and T. Koshikawa, Phys.: Condens. Matter. 25, 395005 (2013).

[26] N. Ogawa, W. Koshibae, A. J. Beekman, N. Nagaosa, M. Kubota, M. Kawasaki, and Y. Tokura, PNAS 112, 8977 (2015).

[27] C. Banerjee, P. Gruszecki, J. W. Klos, O. Hellwig, M. Krawczyk, and A. Barman, Phys. Rev B 96, 024421 (2017).

[28] I. S. Camara, S. Tacchi, L. -C. Garnier, M. Eddrief, F. Fortuna, G. Carlotti, and M. Marangolo, J. Phys.: Condens. Matter 29, 465803 (2017).

[29] L. Landau and E. Lifshitz, Phys. Z. Sowjetunion 8, 153 (1935).

[30] C. Kittel, Phys. Rev. 70, 965 (1946).

[31] C. Kooy and U. Enz, Philips Res. Rep. 15, 7 (1960).

[32] M. W. Muller, Phys. Rev. 122, 1485 (1961); W. F. Brown, Jr., ibid. 124, 1348 (1961).

[33] A. A. Thiele, Bell Syst. Tech. J. 48, 3287 (1969).

[34] A. H. Bobeck and E. Della Torre, Magnetic Bubbles (North-Holland Publishing Company, Amsterdam, 1975).

[35] J. Davies, R. Clover, B. Lieberman, and D. Rose, IEEE Trans. Magn. 16, 1106 (1980).

[36] P. Pavan, R. Bez, P. Olivo, and E. Zanoni, Proc. IEEE 85, 8 (1997).

[37] P. Molho, J. L. Porteseil, Y. Souche, J. Gouzerh, and J. C. S. Levy, J. Appl. Phys. 61, 4188 (1987).

[38] M. Seul and C. A. Murray, Science 262, 558 (1993).

[39] M. Seul and D. Andelman, Science 267, 476 (1995).

[40] H. Nakajima, A. Kotani, K. Harada, Y. Ishii, and S. Mori, Phys. Rev. B 94, 224427 (2016).

[41] L. Sun, R. X. Cao, B. F. Miao, Z. Feng, B. You, D. Wu, W. Zhang, An Hu, and H. F. Ding, Phys. Rev. Lett. 110, 167201 (2013). 
[42] W. B. Zeper, F. J. A. M. Greidanus, P. F. Carcia, IEEE Trans. Magn. 25, 3764 (1989).

[43] W. B. Zeper, H. W. van Kesteren, B. A. J. Jacobs, J. H. M. Spruit, and P. F. Carcia, J. Appl. Phys. 70, 2264 (1991).

[44] Y. Yafet and E. M. Gyorgy, Phys. Rev. B 38, 9145 (1988).

[45] F. J. A. den Broeder, D. Kuiper, A. P. van de Mosselaer, and W. Hoving, Phys. Rev. Lett. 60, 2769 (1988).

[46] C. -J. Lin, G. L. Gorman, C. H. Lee, R. F. C. Farrow, E. E. Marinero, H. V. Do, H. Notarys, and C. J. Chien, J. Magn. Magn. Mat. 93, 194-206 (1991).

[47] M. T. Johnson, P. J. H. Bloemen, F. J. A. den Broeder and J. J. de Vriest, Rep. Prog. Phys. 59, 1409 (1996).

[48] J. Gao, S. Tang, Y. Li, W. Xia, T. Tang, and Y. Du., J. of Appl. Phys. 112, 073913 (2012).

[49] K. Chesnel, A. S. Westover, C. Richards, B. Newbold, M. Healey, L. Hindman, B. Dodson, K. Cardon, D. Montealegre, J. Metzner, F. Samad, L. Fallarino, B. Böhm, T. Schneider, and O. Hellwig, accepted in Phys. Rev. B.

[50] A. Westover, K. Chesnel, K. Hatch, P. Salter, and O. Hellwig, J. Magn. Magn. Mater. 399, 164 (2016).

[51] N. Nakajima, T. Koide, T. Shidara, H. Miyauchi, H. Fukutani, A. Fujimori, K. Iio, T. Katayama, M. Nývlt, and Y. Suzuki, Phys. Rev. Lett. 81, 5229 (1998).

[52] B. N. Engel, C. D. England, R. A. Van Leeuwen, M. H. Wiedmann, and C. M. Falco, Phy. Rev. Lett. 67, 1910 (1991).

[53] J.-W. Lee, J.-R. Jeong, S.-C. Shin, J. Kim, S.-K. Kim, Phys. Rev. B 66, 172409 (2002).

[54] M. Kisielewski, A. Maziewski, M. Tekielak, J. Ferré, S. Lemerle, V. Mathet, and C. Chappert, J. Magn. Magn. Mat. 260, 231-243 (2003).

[55] R. Fromter, H. Stillrich, C. Menk, and H. P. Oepen, Phys. Rev. Lett. 100, 207202 (2008).

[56] Z. Malek, V. Kambersky, Czech. J. Phys. 8, 416 (1958).

[57] R. Allenspach and M. Stampanoni, in Magnetic Surfaces, Thin Films, and Multilayers, edited by S. S. P. Parkin et al., MRS Symposia Proceedings No. 231 (Materials Research Society, Pittsburgh, 1992), p.17.

[58] W. Jiang, G. Chen, K. Liu, J. Zang, S. G. E. te Velthuis, and A. Hoffmann, Phys. Rep. 704, 1 (2017).

[59] M. Birkholz, Thin Film Analysis by X-Ray Scattering (Wiley, New York, 2006). 
[60] O. Hellwig, S. Maat, J. B. Kortright, and E. E. Fullerton, Phys. Rev. B 65, 144418 (2002).

[61] O. Hellwig, G. P. Denbeaux, J. B. Kortright, and E. E. Fullerton, Physica B 336, 136 (2003).

[62] A. Berger, A. W. Pang, and H. Hopster, Phys. Rev. B 52, 1078 (1995).

[63] See Supplemental Material at [URL will be inserted by publisher] for the determination of room temperature saturation magnetization $M_{S}$ and anisotropy $K_{u}$, and the equations used to calculate the stripe domain period data as well as to fit the IP saturation fields as a function of $N$.

[64] L. Fallarino, B. J. Kirby, M. Pancaldi, P. Riego, A. L. Balk, C. W. Miller, P. Vavassori, and A. Berger, Phys. Rev. B 95,134445 (2017).

[65] L. Fallarino, P. Riego, B. J. Kirby, C. W. Miller, and A. Berger, Materials 11, 251 (2018).

[66] S. Tacchi, S. Fin, G. Carlotti, G. Gubbiotti, M. Madami, M. Barturen, M. Marangolo, M. Eddrief, D. Bisero, A. Rettori, and M. G. Pini, Phys. Rev. B 89, 024411 (2014).

[67] J. Muller, J. Appl. Phys. 38, 2413 (1967).

[68] N. Saito, H. Fujiwara, and Y. Sugita, J. Phys. Soc. Jpn. 19, 1116 (1964).

[69] E. Sallica Leva, R. C. Valente, F. Martinez Tabares, M. Vasquez Mansilla, S. Roshdestwensky, and A. Butera, Phys. Rev. B 82, 144410 (2010).

[70] S. A. Montoya, S. Couture, J. J. Chess, J. C. T. Lee, N. Kent, M. -Y. Im, S. D. Kevan, P. Fischer, B. J. McMorran, S. Roy, V. Lomakin, and E. E. Fullerton, Phys. Rev. B 95, 224405 (2017). [71] S. A. Montoya, S. Couture, J. J. Chess, J. C. T. Lee, N. Kent, D. Henze, S. K. Sinha, M. -Y. Im, S. D. Kevan, P. Fischer, B. J. McMorran, V. Lomakin, S. Roy, and E. E. Fullerton, Phys. Rev. B 95, 024415 (2017).

[72] Y. Murayama, J. Phys. Soc. Jpn. 21, 2253 (1966).

[73] B. Kaplan and G. A. Gehring, J. Magn. Magn. Mat. 128, 111 (1993).

[74] S. H. Charap and J. M. Nemchik, IEEE Tran. Magn. 5, 566 (1969).

[75] J. A. Cape and G. W. Lehman, J. Appl. Phy. 42, 5732 (1971).

[76] F. W. F. Dorleijn, W. F. Druyvesteyn, F. A. de Jonge, and H. M. W. Boolij, IEEE Trans. Magn. 7, 355-358 (1971).

[77] S. Woo, K. Litzius, B. Krüger, M.-Y. Im, L. Caretta, K. Richter, M. Mann, A. Krone, R. M. Reeve, M. Weigand, P. Agrawal, I. Lemesh, M.-A. Mawass, P. Fischer, M. Kläui, and G. S. D. Beach, Nat. Mater. 15, 501 (2016).

[78] S.-G. Je, D.-H. Kim, S.-C. Yoo, B.-C. Min, K.-J. Lee, and S.-B. Choe, Phys. Rev. B 88, 214401 (2013). 
[79] S. Neusser and D. Grundler, Adv. Mater. 21, 2927 (2009).

[80] H. Yang, A. Thiaville, S. Rohart, A. Fert, and M. Chshiev, Phys. Rev. Lett. 115, 267210 (2015).

[81] A. Soumyanarayanan, M. Raju, A. L. Gonzalez Oyarce, A. K. C. Tan, M.-Y. Im, A. P. Petrovic, P. Ho, K. H. Khoo, M. Tran, C. K. Gan, F. Ernult, and C. Panagopoulos, Nat. Mater. 16, 898 (2017). 